International Journal of Bifurcation and Chaos, Vol. 10, No. 1 (2000) 1-23

(c) World Scientific Publishing Company

\title{
SIMPLEST ODE EQUIVALENTS OF CHUA'S EQUATIONS
}

\author{
JIŘÍ POSPÍŠIL, ZDENĚK KOLKA, \\ JANA HORSKÁ and JAROMÍR BRZOBOHATÝ* \\ Institute of Radio Electronics, \\ ${ }^{*}$ Institute of Microelectronics, \\ Faculty of Electrical Engineering and Computer Sciences, \\ Brno University of Technology, \\ Purkyñova 118, 612 00 Brno, Czech Republic
}

Received March 20, 1999; Revised September 30, 1999

\begin{abstract}
The so-called elementary canonical state models of the third-order piecewise-linear (PWL) dynamical systems, as the simplest ODE equivalents of Chua's equations, are presented. Their mutual relations using the linear topological conjugacy are demonstrated in order to show in detail that Chua's equations and their canonical ODE equivalents represent various forms of qualitatively equivalent models of third-order dynamical systems. New geometrical aspects of the corresponding transformations together with examples of typical chaotic attractors in the stereoscopic view, give the possibility of a deeper insight into the third-order system dynamics.
\end{abstract}

\section{Introduction}

The state representation of dynamical systems is currently used in diverse fields of science and engineering. Its simple, well arranged and transparent form expressed as a compact set of first-order ordinary differential equations (ODE) is also very convenient for modeling chaotic systems considered as determined systems exhibiting complex and unpredictable behavior.

Many paradigms of chaotic systems modeling the main features of chaotic dynamics with only a few nonlinear equations, e.g. the Duffing equation, the Lorenz system, the Rössler system, Chua's equations, etc., are already known. Among all these paradigms is the Chua's model, i.e. Chua's equations and the corresponding Chua's circuits (Fig. 1), that is of unique significance and for several reasons represents a milestone in the research on chaos theory and application. In comparison with the previous paradigms, Chua's model has the following advantages [Liao \& Chen, 1998] (i) It uses only one nonlinearity with one variable so that the same model can be used with any scalar nonlinearity, i.e. also any piecewise-linear (PWL) function.

(ii) It can be easily implemented using currently used electronic components (Fig. 1).

(iii) It possesses an explicit Poincaré's map that can be derived and proved by rigorous mathematical methods.

The main results associated with Chua's circuits/equations have already been summarized in two special issues of the Journal of Circuits, Systems, and Computers [Madan, 1993a, 1993b] and also in two fundamental papers [Chua et al., 1993a, 1993b] published in the "Special Issue on Chaos in Nonlinear Electronic Circuits - Part A. Tutorial and Reviews" of the IEEE Transactions on Circuits and Systems. For more details published in many other papers and for a complete survey of the problem, the reader is referred to the chronological bibliographies in all these Special 


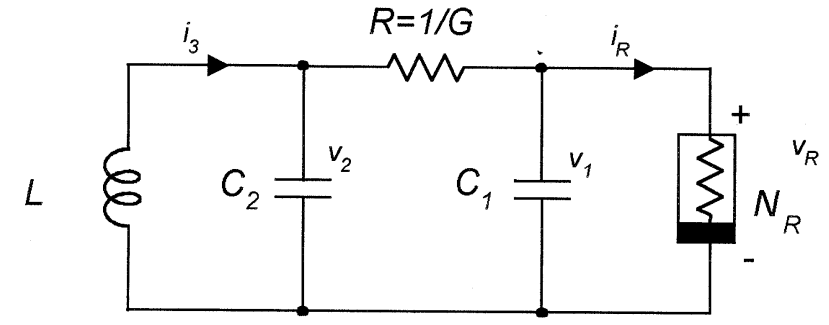

(a)

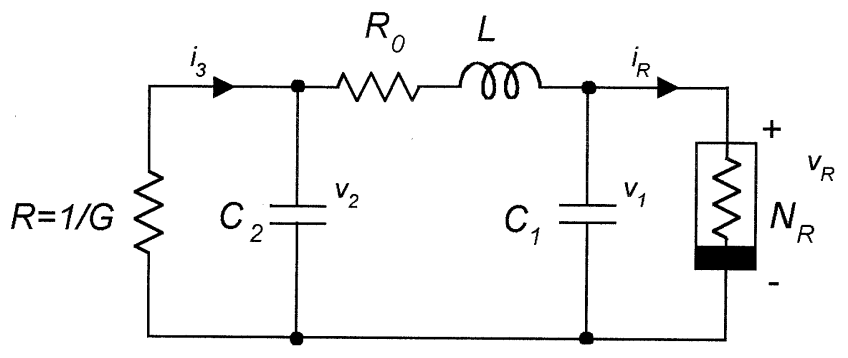

(b)

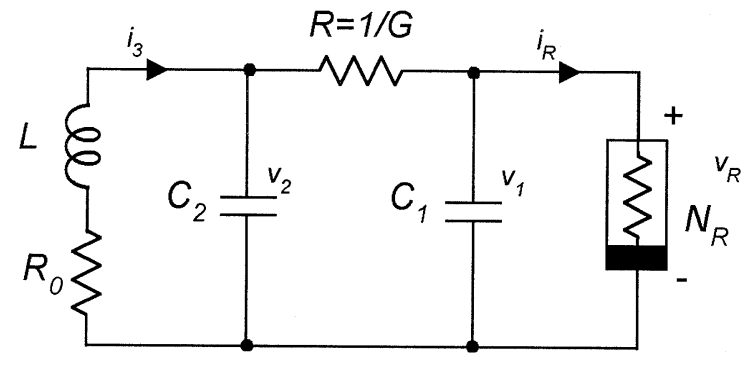

(c)

Fig. 1. Chua's circuit family. (a) Original (noncanonical) Chua's circuit. (b) Universal (canonical) Chua's circuit. (c) Chua's oscillator.

Issues and also in the fundamental paper [Chua, 1993].

Chua's circuit in its original (noncanonical) form, shown in Fig. 1(a), was synthesized to form the simplest third-order autonomous electronic generator of chaotic signals. Its chaotic nature was verified by computer simulation [Matsumoto, 1984], investigated experimentally [Zhong \& Ayrom, 1985] and confirmed also by a rigorous mathematical proof [Chua et al., 1986]. The history of this creative research period, including all the dramatic circumstances, is vividly described in [Chua, 1992]. These remarkable results have initiated among engineers, mathematicians, and physicists intensive worldwide research activities in this field. Later many aspects of Chua's circuit/equations have been studied, the previous

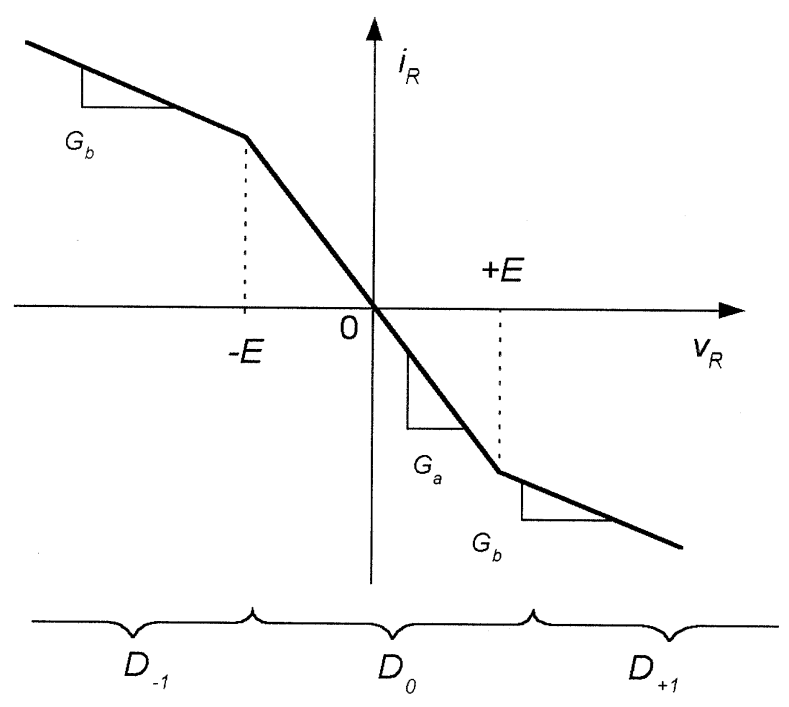

(a)
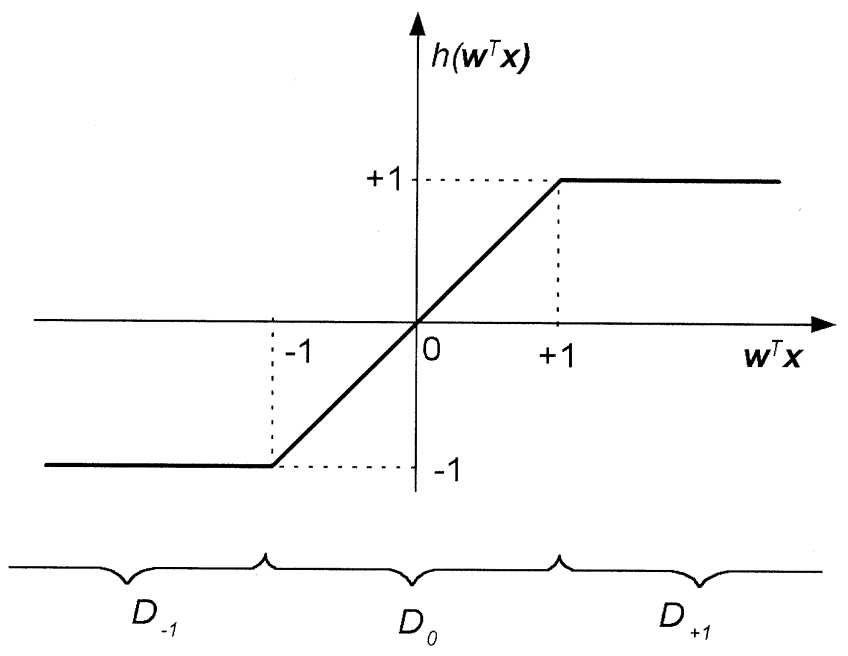

(b)

Fig. 2. Three-region odd-symmetric piecewise-linear (PWL) functions. (a) Typical $v_{R}-i_{R}$ characteristic of Chua's diode. (b) Elementary memoryless saturation function.

results have been completed, extended, generalized, and then applied in many areas of science and engineering. Several selected papers quoted in the following paragraph suggest various directions of this investigation and some of the results achieved.

Two generalized circuits shown in Fig. 1(b) [Chua \& Lin, 1990] and in Fig. 1(c) [Chua, 1993] eliminate the eigenvalue constraints of the original circuit caused by the missing free parameter realized here by inserting a linear resistor $R_{0}$. Because of the close relation to original Chua's circuit, its unfolded and canonical form called Chua's oscillator [Fig. 1(c)] has become the 
universal paradigm for chaos. The nonlinear resistor $N_{R}$ (so-called Chua's diode), first realized in discrete form [Kennedy, 1992], has also been designed in IC form [Cruz \& Chua, 1992] as a more convenient device for industrial applications. Its $v_{R}-i_{R}$ characteristic considered for simplicity as a PWL odd-symmetric function shown in Fig. 2(a) has been substituted by various nonlinear smooth functions, e.g. polynomial [Zhong, 1994; Huang et al., 1996], sigmoid and signum [Brown, 1993], etc. Using Chua's oscillator many familiar chaotic circuits and systems belonging to the family of vector field $C \backslash C_{0}^{1}$ have been modeled and numerically simulated [Chua, 1993; Lindberg, 1993], and a special PC-based software package has been developed for Chua's circuit family simulation by M. P. Kennedy [Chua et al., 1993b]. New attractor types have been found [Belykh \& Chua, $1992]$ and various routes to chaos have been demonstrated using this paradigm [Chua et al., 1993a; Kennedy, 1993a, 1993b]. Its realization using several circuit techniques, including the CNN [Zou \& Nossek, 1993; Arena et al., 1995] and integrators [Delgado-Restituto \& Rodriguez-Vasquez, 1998], has been designed. Chaotic regions of Chua's circuits/equations have been investigated [Ogorzalek, 1987] as well as the range of bifurcation [Nasser et al., 1993; Huang, 1996] and boundary surfaces [Pivka \& Špány, 1993]. Chua's oscillator has also been applied to many problems of controlled and synchronized chaos and its utilization [Ogorzalek, 1993a, 1993b; Hasler, 1994; Feldmann et al., 1996; Liao \& Chen, 1998], etc. Chua's equations have been generalized for modeling the Rössler and the Lorenz dynamics [Brown, 1993] and also for higher-order systems [Kočarev et al., 1993; Schwarz et al., 1993]. To determine the mutual relations between Chua's oscillator and other third-order dynamical systems belonging to Class $C$ of vector field in $\Re^{3}$ the theorem of the linear topological conjugacy has been utilized in its special form [Chua, 1993], and the concept of qualitative equivalence has been defined.

One of the new theoretical approaches starting from the previous research is the so-called "elementary" canonical state model of Chua's circuit family [Pospíšil \& Brzobohatý, 1996a]. Its two basic forms are linearly conjugate to Chua's oscillator and represent the simplest ODE equivalents of Chua's equations. Same as Chua's oscillator, they are canonical with respect to the behavior, i.e. capable of realizing all possible behavior of the associated vector field, and with respect to the number of equation/circuit parameters, i.e. containing the minimum number of elements necessary [Schwarz et al., 1993]. In addition, they have an elementary relation between their equation parameters and the equivalent eigenvalue parameters. ${ }^{1}$ Such a property can be useful for both theoretical and practical studies of chaos. Both these dual canonical $^{2}$ ODE forms and Chua's equations therefore represent various forms of qualitatively equivalent models of third-order autonomous dynamical systems.

The paper [Pospíšil \& Brzobohatý, 1996a] contains only the basic definitions of both canonical ODE forms and brief information about their mutual relation expressed by the generalized linear topological conjugacy. The connection with Chua's equations is suggested only in general terms and its detailed form has not been published yet. In this paper all the previous results are summarized, completed, and extended by details of mutual transformations among all these qualitatively equivalent ODE forms. In Sec. 2, the basic properties of all these models as well as their formal comparison are given. In Sec. 3, the detailed forms of their mutual linear topological conjugacy, including graphical illustrations, are presented. In Sec. 4, the stereoscopic color gallery of several typical chaotic attractors represented by the first canonical ODE form is shown. Section 5 contains conclusions and comments on the latest results achieved and on what other results can be expected.

\section{Systems of Class $C$ and Their ODE Equivalents}

To determine Class $C$ of vector fields in $\Re^{3}$ and its basic properties the theoretical preliminaries are first summarized. Then the three basic ODE equivalents, i.e. Chua's equations and the two canonical forms, are defined. Finally, their basic properties, formal relations and a set of simple

\footnotetext{
${ }^{1}$ Definition and other details are given in Sec. 2.

${ }^{2}$ In the theory of linear systems the adjective "canonical" denotes the models/systems having all these three properties including the elementary parameter relation mentioned. In accordance with this terminology, the same simple designation is subsequently also used for both these new ODE forms, i.e. without specifying "elementary".
} 
trajectories demonstrating their similar dynamical behavior are shown.

\subsection{Systems of Class C}

Definition. A dynamical system, circuit or vector field defined by state equation

$$
\dot{\mathbf{x}}=\mathbf{f}(\mathbf{x}), \quad \mathbf{x} \in \Re^{3}
$$

is said to belong to Class $C$ iff the following three conditions are valid:

(i) $\mathbf{f}(\cdot)$ is continuous

(ii) $\mathbf{f}(\cdot)$ is odd-symmetric, i.e. $\mathbf{f}(\mathbf{x})=-\mathbf{f}(-\mathbf{x})$

(iii) $\Re^{3}$ is partitioned by two parallel boundary planes $U_{1}$ and $U_{-1}$ into an inner region $D_{0}$ and two outer regions $D_{1}$ and $D_{-1}$, and $\mathbf{f}(\cdot)$ is affine in each region.

The boundary planes $U_{1}$ and $U_{-1}$ can generally be chosen to be

$$
\begin{array}{cl}
U_{1}: & \mathbf{w}^{\mathrm{T}} \mathbf{x}=1 \\
U_{-1}: & \mathbf{w}^{\mathrm{T}} \mathbf{x}=-1
\end{array}
$$

where vectors $\mathbf{w}$ and $\mathbf{x}$ are

$$
\mathbf{w}=\left(\begin{array}{c}
w_{1} \\
w_{2} \\
w_{3}
\end{array}\right), \quad \mathbf{x}=\left(\begin{array}{c}
x \\
y \\
z
\end{array}\right)
$$

so that vector dot product $\mathbf{w}^{\mathrm{T}} \mathbf{x}$ represents a linear combination of variables $x, y, z$, i.e.

$$
\mathbf{w}^{\mathrm{T}} \mathbf{x}=w_{1} x+w_{2} y+w_{3} z .
$$

Then any vector field in Class $C$ can be expressed as

$$
\begin{array}{ll}
\dot{\mathbf{x}}=\mathbf{A} \mathbf{x}+\mathbf{b}, \quad \mathbf{w}^{\mathrm{T}} \mathbf{x} \geq 1 \\
\dot{\mathbf{x}}=\mathbf{A} \mathbf{x}-\mathbf{b}, \quad \mathbf{w}^{\mathrm{T}} \mathbf{x} \leq-1 \\
\dot{\mathbf{x}}=\mathbf{A}_{0} \mathbf{x}, \quad-1 \leq \mathbf{w}^{\mathrm{T}} \mathbf{x} \leq 1
\end{array}
$$

where state matrix $\mathbf{A}$ and vector $\mathbf{b}$ are

$$
\mathbf{A}=\left[\begin{array}{lll}
a_{11} & a_{12} & a_{13} \\
a_{21} & a_{22} & a_{23} \\
a_{31} & a_{32} & a_{33}
\end{array}\right], \quad \mathbf{b}=\left[\begin{array}{l}
b_{1} \\
b_{2} \\
b_{3}
\end{array}\right]
$$

and define an affine vector field in outer regions $D_{1}$ and $D_{-1}$. Because of continuity, matrix $\mathbf{A}_{0}$ is generally given as

$$
\begin{aligned}
\mathbf{A}_{0} & =\mathbf{A}+\mathbf{b w}^{\mathrm{T}} \\
& =\left[\begin{array}{lll}
a_{11}+b_{1} w_{1} & a_{12}+b_{1} w_{2} & a_{13}+b_{1} w_{3} \\
a_{21}+b_{2} w_{1} & a_{22}+b_{2} w_{2} & a_{23}+b_{2} w_{3} \\
a_{31}+b_{3} w_{1} & a_{32}+b_{3} w_{2} & a_{33}+b_{3} w_{3}
\end{array}\right]
\end{aligned}
$$

and defines a linear vector field in inner region $D_{0}$.

It is easy to show that Eqs. (4)-(6) can be recast into the equivalent compact explicit form

$$
\dot{\mathbf{x}}=\mathbf{A} \mathbf{x}+\mathbf{b} h\left(\mathbf{w}^{\mathrm{T}} \mathbf{x}\right)
$$

where $h\left(\mathbf{w}^{\mathrm{T}} \mathbf{x}\right)$ represents the elementary threesegment odd-symmetric PWL function [Fig. 2(b)]

$$
h\left(\mathbf{w}^{\mathrm{T}} \mathbf{x}\right)=\frac{1}{2}\left(\left|\mathbf{w}^{\mathrm{T}} \mathbf{x}+1\right|-\left|\mathbf{w}^{\mathrm{T}} \mathbf{x}-1\right|\right)
$$

Note that $h\left(\mathbf{w}^{\mathrm{T}} \mathbf{x}\right)= \pm 1$ for $\left|\mathbf{w}^{\mathrm{T}} \mathbf{x}\right| \geq 1$ and Eq. (7) reduces either to Eq. (4a) or to Eq. (4b). Similarly when $\left|\mathbf{w}^{\mathrm{T}} \mathbf{x}\right| \leq 1$ then $h\left(\mathbf{w}^{\mathrm{T}} \mathbf{x}\right)=\mathbf{x}$ and Eq. (7) reduces to Eq. (4c), where matrix $\mathbf{A}_{0}$ is given by Eq. (6). ${ }^{3}$

The dynamical behavior of the system described by Eqs. (7) and (8) is defined by the two sets of eigenvalues $\left\{\mu_{1}, \mu_{2}, \mu_{3}\right\}$ and $\left\{\nu_{1}, \nu_{2}, \nu_{3}\right\}$, determining two characteristic polynomials associated with the Jacobian matrix in the corresponding regions, i.e.

$$
\begin{aligned}
D_{0}: P(s) & =\operatorname{det}\left(s \mathbf{1}-\mathbf{A}_{0}\right) \\
& =\left(s-\mu_{1}\right)\left(s-\mu_{2}\right)\left(s-\mu_{3}\right) \\
& =s^{3}-p_{1} s^{2}+p_{2} s-p_{3}, \\
D_{1}, D_{-1}: Q(s) & =\operatorname{det}(s \mathbf{1}-\mathbf{A}) \\
& =\left(s-\nu_{1}\right)\left(s-\nu_{2}\right)\left(s-\nu_{3}\right) \\
& =s^{3}-q_{1} s^{2}+q_{2} s-q_{3}
\end{aligned}
$$

where $\mathbf{1}$ is the unity matrix. The coefficients of the characteristic polynomials (the so-called equivalent eigenvalue parameters) can be expressed as

\footnotetext{
${ }^{3}$ Similar derivation has been used for the special case $\mathbf{w}^{\mathrm{T}}=\left(\begin{array}{lll}1 & 0 & 0\end{array}\right)$ in the papers [Chua, 1993] and [Chua et al., 1993a, $1993 \mathrm{~b}]$.
} 


$$
\begin{array}{ll}
p_{1}=\mu_{1}+\mu_{2}+\mu_{3} & q_{1}=\nu_{1}+\nu_{2}+\nu_{3} \\
p_{2}=\mu_{1} \mu_{2}+\mu_{2} \mu_{3}+\mu_{1} \mu_{3} & q_{2}=\nu_{1} \nu_{2}+\nu_{2} \nu_{3}+\nu_{1} \nu_{3} \\
p_{3}=\mu_{1} \mu_{2} \mu_{3} & q_{3}=\nu_{1} \nu_{2} \nu_{3}
\end{array}
$$

These parameters are more convenient to work with than the eigenvalues because they are real numbers whereas the eigenvalues may be complex numbers. As follows from Eqs. (9)-(11) a minimum of six free parameters must be available in any ODE equivalent for complete determination of the dynamical behavior of Class $C$. Note that the latter does not depend on the positions of two parallel boundary planes $U_{1}$ and $U_{-1}$ so that, without a loss of generality, the unity breakpoint values $\mathbf{w}^{\mathrm{T}} \mathbf{x}= \pm 1$ used in Eqs. (2)-(8) can be accepted.

In the next subsections the three different forms (i.e. basic, modified, and global) of all the ODE equivalents considered, i.e. Chua's equations and both canonical models, are presented. To demonstrate their properties and to simplify their comparison the complete state equations and the corresponding integrator-based block diagram for each model are given.

\subsection{Basic forms of $O D E$ equivalents}

\subsubsection{Chua's equations}

Consider the dimensionless form of Chua's equations [Chua et al., 1993a, 1993b], i.e.

$$
\begin{aligned}
\dot{x} & =\frac{d x}{d \tau}=k \alpha(y-x-f(x)) \\
\dot{y} & =\frac{d y}{d \tau}=k(x-y+z) \\
\dot{z} & =\frac{d z}{d \tau}=k(-\beta y-\gamma z) \\
f(x) & =b x+\frac{1}{2}(a-b)(|x+1|-|x-1|)
\end{aligned}
$$

Voltages $v_{1}, v_{2}$ and current $i_{3}$ in Chua's oscillator [Fig. 1(c)] are here transformed into dimensionless variables $x, y, z$ defined as

$$
x \triangleq \frac{v_{1}}{E}, \quad y \triangleq \frac{v_{2}}{E}, \quad z \triangleq i_{3}\left(\frac{R}{E}\right)
$$

where $E$ represents the breakpoint voltage of the PWL Chua's diode [Fig. 2(a)]. Similarly the set of seven real circuit parameters
$\left\{C_{1}, C_{2}, L, R, R_{0}, G_{a}, G_{b}\right\}$ is transformed into the set of six independent dimensionless parameters $\{\alpha, \beta, \gamma, a, b, k\}$ defined as

$$
\begin{aligned}
& \alpha \triangleq \frac{C_{2}}{C_{1}}, \quad \beta \triangleq \frac{R^{2} C_{2}}{L}, \quad \gamma \triangleq \frac{R R_{0} C_{2}}{L} \\
& a \triangleq R G_{a}, \quad b \triangleq R G_{b}, \quad k=\operatorname{sgn}\left(R C_{2}\right)
\end{aligned}
$$

Due to the scaling in time given by

$$
\tau \triangleq \frac{t}{\left|R C_{2}\right|}
$$

the eigenvalues of the dimensionless form are normalized. Other details can be found in two fundamental papers [Chua et al., 1993a, 1993b].

Dimensionless Chua's equations represent the general, compact, and universal ODE form suitable for the comparison with other ODE equivalents. For such a purpose it is convenient to rewrite Eq. (12) into the basic explicit form given by Eq. (7), i.e.

$$
\begin{aligned}
\dot{x} & =-k \alpha(b+1) x+k \alpha y+k \alpha(b-a) h(x) \\
\dot{y} & =k x-k y+k z \\
\dot{z} & =-k \beta y-k \gamma z \\
h(x) & =\frac{1}{2}(|x+1|-|x-1|)
\end{aligned}
$$

so that state matrix $\mathbf{A}$ is

$$
\mathbf{A}=\left[\begin{array}{ccc}
-k \alpha(b+1) & k \alpha & 0 \\
k & -k & k \\
0 & -k \beta & -k \gamma
\end{array}\right]
$$

and vectors $\mathbf{b}, \mathbf{w}$ are

$$
\mathbf{b}=\left(\begin{array}{c}
k \alpha(b-\alpha) \\
0 \\
0
\end{array}\right), \quad \mathbf{w}=\left(\begin{array}{l}
1 \\
0 \\
0
\end{array}\right)
$$

Utilizing Eq. (6) matrix $\mathbf{A}_{0}$ is obtained in a similar form as state matrix $\mathbf{A}$, where parameter $b$ is replaced by parameter $a$, i.e.

$$
\mathbf{A}_{0}=\left[\begin{array}{ccc}
-k \alpha(a+1) & k \alpha & 0 \\
k & -k & k \\
0 & -k \beta & -k \gamma
\end{array}\right]
$$


After substituting $\mathbf{A}_{0}$ from Eq. (17c) into Eq. (9) and $\mathbf{A}$ from Eq. (17a) into Eq. (10) the two sets of the equivalent eigenvalue parameters associated

$$
\begin{aligned}
& p_{1}=-k[\alpha(a+1)+\gamma+1] \\
& p_{2}=a \alpha(\gamma+1)+\gamma(\alpha+1)+\beta \\
& p_{3}=-k \alpha[a(\beta+\gamma)+\beta]
\end{aligned}
$$

To model any system of Class $C$ by Chua's equations it is necessary to derive the inverse form of Eq. (18), i.e. to express the set of dimensionless parameters $\{\alpha, \beta, \gamma, a, b, k\}$ by using equivalent eigenvalue parameters $\left\{p_{1}, p_{2}, p_{3} ; q_{1}, q_{2}, q_{3}\right\}$. The resultant formulas are: ${ }^{4}$

$$
\begin{aligned}
\alpha & =\frac{1}{l_{1} l_{3}^{2}} \\
\beta & =1+\frac{p_{3}-q_{3}}{p_{1}-q_{1}} \frac{1}{l_{1} l_{3}^{2}}+\frac{p_{2}-q_{2}}{p_{1}-q_{1}} \frac{1}{l_{1} l_{3}} \\
\gamma & =-1-\frac{p_{2}-q_{2}}{p_{1}-q_{1}} \frac{1}{l_{1} l_{3}} \\
\alpha & =-1+\left(\frac{p_{2}-q_{2}}{p_{1}-q_{1}}-p_{1}\right) l_{3} \\
b & =-1+\left(\frac{p_{2}-q_{2}}{p_{1}-q_{1}}-q_{1}\right) l_{3} \\
k & =\operatorname{sign}\left(l_{1} l_{3}\right)
\end{aligned}
$$

where

$$
\begin{aligned}
& l_{1}=-p_{2}-\left(\frac{p_{2}-q_{2}}{p_{1}-q_{1}}-p_{1}\right) \frac{p_{2}-q_{2}}{p_{1}-q_{1}}+\frac{p_{3}-q_{3}}{p_{1}-q_{1}} \\
& l_{2}=-p_{3}-\left(\frac{p_{2}-q_{2}}{p_{1}-q_{1}}-p_{1}\right) \frac{p_{3}-q_{3}}{p_{1}-q_{1}} \\
& l_{3}=-\frac{p_{2}-q_{2}}{p_{1}-q_{1}} \frac{1}{l_{1}}-\frac{l_{2}}{l_{1}^{2}}
\end{aligned}
$$

The corresponding integrator-based block diagram can be derived directly from Eq. (16). It contains three ideal invertors, three adders, and one PWL feedback block as shown in Fig. 3(a). Note that such a network representation is not typical of or natural for Chua's equations and it is used here only to provide a complete survey and better comparison with other ODE equivalents.

\footnotetext{
${ }^{4}$ Other details are given in [Chua et al., 1993a, 1993b].

${ }^{5}$ The formal proof is given in [Pospísil \& Brzobohatý, 1996a].
}

with the corresponding regions can be expressed by using dimensionless parameters $\{\alpha, \beta, \gamma, a, b, k\}$ as follows

$$
\begin{aligned}
& q_{1}=-k[\alpha(b+1)+\gamma+1] \\
& q_{2}=b \alpha(\gamma+1)+\gamma(\alpha+1)+\beta \\
& q_{3}=-k \alpha[b(\beta+\gamma)+\beta]
\end{aligned}
$$

\subsubsection{First canonical ODE equivalent}

The complete set of state equations for this model introduced in [Pospíšil \& Brzobohatý, 1996a] has the following form

$$
\begin{aligned}
& \dot{x}=q_{1} x-y+\left(p_{1}-q_{1}\right) h(x) \\
& \dot{y}=q_{2} x-z+\left(p_{2}-q_{2}\right) h(x) \\
& \dot{z}=q_{3} x+\left(p_{3}-q_{3}\right) h(x) \\
& h(x)=\frac{1}{2}(|x+1|-|x-1|)
\end{aligned}
$$

and represents one of the two simplest ODE equivalents of autonomous PWL dynamical third-order systems belonging to Class $C$. Matrices $\mathbf{A}, \mathbf{A}_{0}$ and vectors $\mathbf{b}, \mathbf{w}$ have the elementary forms

$$
\begin{aligned}
\mathbf{A} & =\left[\begin{array}{rrr}
q_{1} & -1 & 0 \\
q_{2} & 0 & -1 \\
q_{3} & 0 & 0
\end{array}\right], \quad \mathbf{b}=\left(\begin{array}{l}
p_{1}-q_{1} \\
p_{2}-q_{2} \\
p_{3}-q_{3}
\end{array}\right), \\
\mathbf{A}_{0} & =\left[\begin{array}{rrr}
p_{1} & -1 & 0 \\
p_{2} & 0 & -1 \\
p_{3} & 0 & 0
\end{array}\right], \quad \mathbf{w}=\left(\begin{array}{l}
1 \\
0 \\
0
\end{array}\right)
\end{aligned}
$$

where the main parameters are identical with the equivalent eigenvalue parameters (except for units and zeros). ${ }^{5}$ The integrator-based block diagram derived directly from Eq. (21) and containing the same number of elements as the previous model is shown in Fig. 3(b). This structure represents the well-known first canonical form of linear nonautonomous dynamical systems (the so-called Analogue Computer Network [Perry, 1975]) completed by the feedback block with elementary PWL function $h(x)$. 


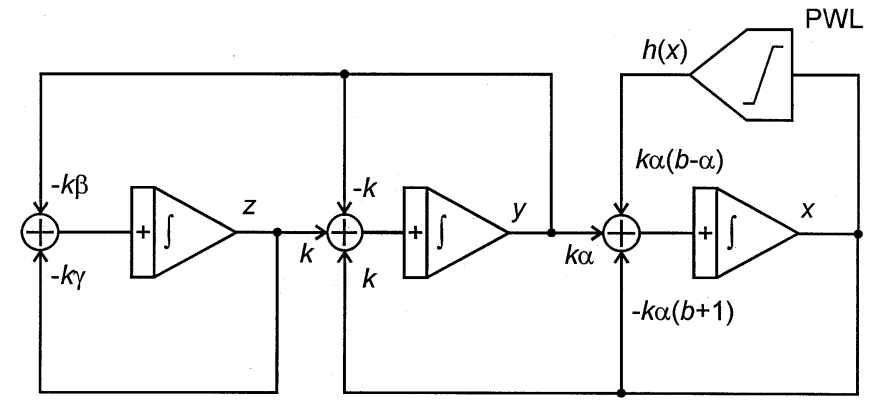

(a)

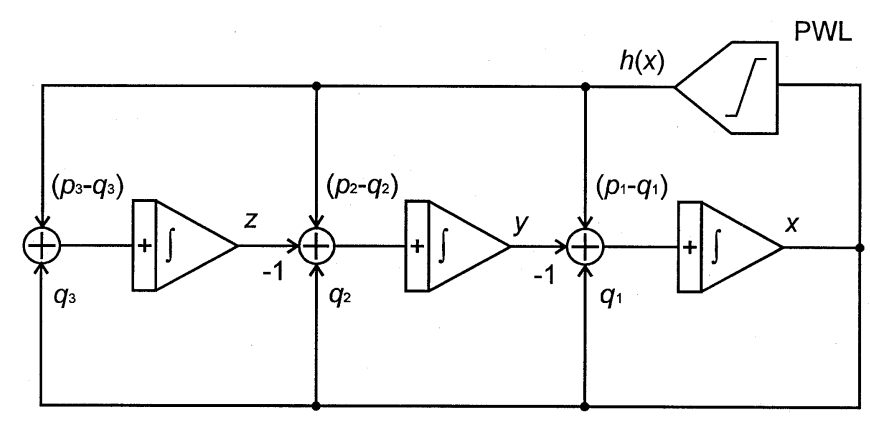

(b)

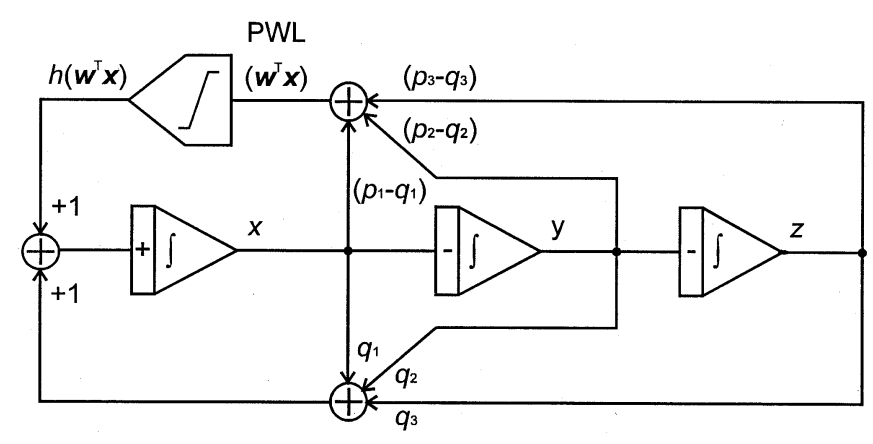

(c)

Fig. 3. Basic forms of integrator-based block diagrams. (a) Chua's equations. (b) First canonical ODE equivalent. (c) Second canonical ODE equivalent.

\subsubsection{Second canonical ODE equivalent}

This model represents a dual case to the first one [Pospísil \& Brzobohatý, 1996a] so that the complete set of state equations is

$$
\begin{aligned}
\dot{x} & =q_{1} x+q_{2} y+q_{3} z+h\left(\mathbf{w}^{\mathrm{T}} \mathbf{x}\right) \\
\dot{y} & =-x \\
\dot{z} & =-y \\
\mathbf{w}^{\mathrm{T}} \mathbf{x} & =\left(p_{1}-q_{1}\right) x+\left(p_{2}-q_{2}\right) y+\left(p_{3}-q_{3}\right) z
\end{aligned}
$$

Matrices $\mathbf{A}, \mathbf{A}_{0}$ and vectors $\mathbf{b}, \mathbf{w}$ also have, therefore, dual forms to Eq. (22), i.e.

$$
\begin{aligned}
& \mathbf{A}=\left[\begin{array}{rrc}
q_{1} & q_{2} & q_{3} \\
-1 & 0 & 0 \\
0 & -1 & 0
\end{array}\right], \quad \mathbf{b}=\left(\begin{array}{l}
1 \\
0 \\
0
\end{array}\right), \\
& \mathbf{A}_{0}=\left[\begin{array}{rrc}
p_{1} & p_{2} & p_{3} \\
-1 & 0 & 0 \\
0 & -1 & 0
\end{array}\right], \quad \mathbf{w}=\left(\begin{array}{l}
p_{1}-q_{1} \\
p_{2}-q_{2} \\
p_{3}-q_{3}
\end{array}\right)
\end{aligned}
$$

where the main parameters are again identical with the equivalent eigenvalue parameters. ${ }^{5}$

The integrator-based block diagram derived directly from Eq. (23) is shown in Fig. 3(c). It contains the same number of blocks, with two of the integrators inverting. This structure represents the well-known second canonical form of linear nonautonomous dynamical systems (the socalled Follow-the-Leader-Feedback Network [Laker \& Ghausi, 1974]) completed by the same feedback block with elementary PWL function $h(x)$.

\subsection{Modified forms of $O D E$ equivalents}

The gains of individual adders in the integratorbased block diagrams shown in Fig. 3 are determined by the corresponding coefficients in Eqs. (16), (21) and (23) and generally they can be a function of several model parameters. To achieve structures where each of the adder gains is given by one parameter only, the basic explicit forms introduced in Sec. 2.2 can be rewritten into the forms with separated parameters as shown in the next paragraphs. These modified equations and the corresponding block diagrams can be useful in all the cases where the influence of individual parameters on the system dynamics has to be investigated, e.g. bifurcation phenomena, sensitivity properties, etc.

\subsubsection{Chua's equations}

The modified Eq. (16) has the following form

$$
\begin{aligned}
\dot{x} & =k \alpha\{(y-x)+b[h(x)-x]-a h(x)\} \\
\dot{y} & =k(x-y+z) \\
\dot{z} & =-k(\beta y+\gamma z) \\
h(x) & =\frac{1}{2}(|x+1|-|x-1|)
\end{aligned}
$$




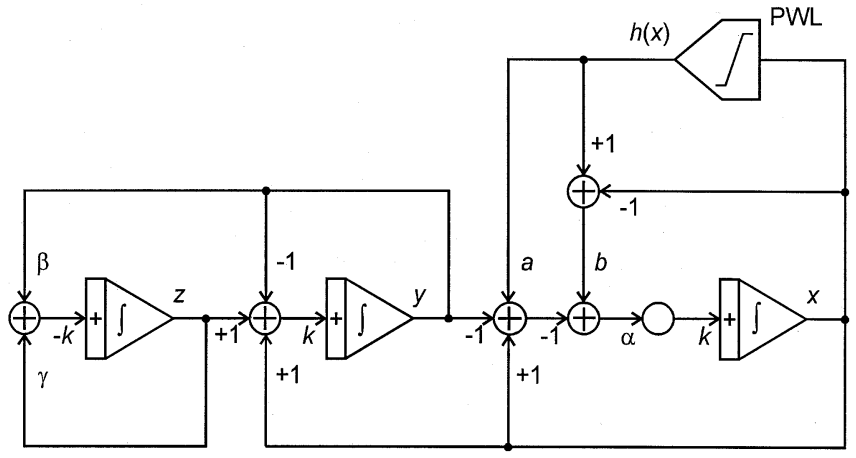

(a)

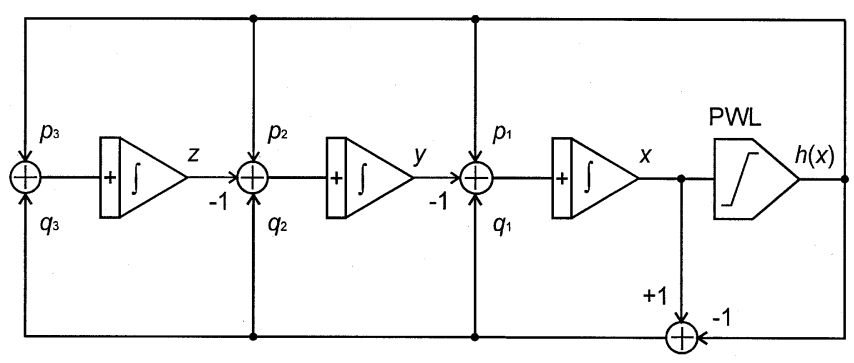

(b)

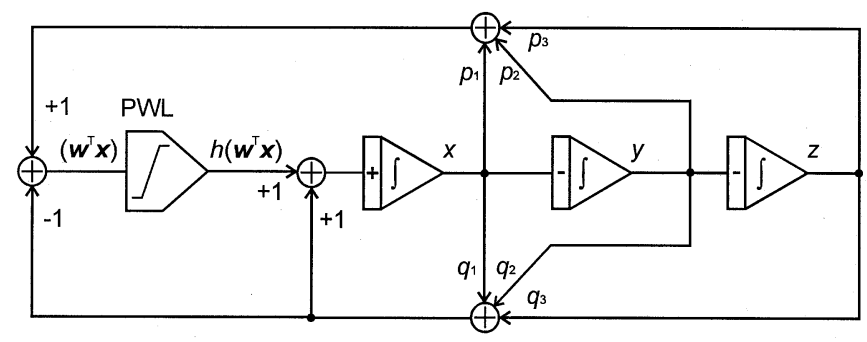

(c)

Fig. 4. Modified forms of integrator-based block diagrams with separated parameters. (a) Chua's equations. (b) First canonical ODE equivalent. (c) Second canonical ODE equivalent.

The corresponding block diagram containing several additional adders is shown in Fig. 4(a). Parameter $k$ determines here the polarity of individual integrators.

\subsubsection{First canonical ODE equivalent}

The modified form of Eq. (21) can be written as

$$
\begin{aligned}
& \dot{x}=p_{1} h(x)+q_{1}[h(x)-x]-y \\
& \dot{y}=p_{2} h(x)+q_{2}[h(x)-x]-z \\
& \dot{z}=p_{3} h(x)+q_{3}[h(x)-x] \\
& h(x)=\frac{1}{2}(|x+1|-|x-1|)
\end{aligned}
$$

The corresponding block diagram containing one additional adder is shown in Fig. 4(b).

\subsubsection{Second canonical ODE equivalent}

Finally, Eq. (23) can be rearranged as

$$
\begin{aligned}
& \dot{x}=\left(q_{1} x+q_{2} y+q_{3} z\right)+h\left(\mathbf{w}^{\mathrm{T}} \mathbf{x}\right) \\
& \dot{y}=-x \\
& \dot{z}=-y \\
& \mathbf{w}^{\mathrm{T}} \mathbf{x}=\left(p_{1} x+p_{2} y+p_{3} z\right)-\left(q_{1} x+q_{2} y+q_{3} z\right)
\end{aligned}
$$

Also in this case, one additional adder models this formal modification as shown in the block diagram in Fig. 4(c).

\subsection{Global forms of ODE equivalents}

The basic forms of ODE equivalents given by Eqs. (16), (21) and (23) can also be rewritten into the so-called global form, where the elementary PWL function $h\left(\mathbf{w}^{\mathrm{T}} \mathbf{x}\right)$ in each equation is completed by a linear part with the same argument $\mathbf{w}^{\mathrm{T}} \mathbf{x}$ so that the total PWL functions $F_{i}\left(\mathbf{w}^{\mathrm{T}} \mathbf{x}\right)$, $i=1,2,3$; are defined. It entails the simplified forms of the corresponding block diagram and provides a "global" view into the system.

\subsubsection{Chua's equations}

Denoting in Eq. (16)

$$
F(x)=-k \alpha[(b+1) x+(a-b) h(x)]
$$

the equation can be rearranged into the form

$$
\begin{aligned}
& \dot{x}=k \alpha y+F(x) \\
& \dot{y}=k(x-y+z) \\
& \dot{z}=-k(\beta y+\gamma z)
\end{aligned}
$$

which is very similar to the original form given by Eq. (12), where $F(x)=-k a[x+f(x)]$ so that the corresponding block diagram shown in Fig. 5(a) is also similar to Fig. 3(a).

\subsubsection{First canonical ODE equivalent}

When denoting in Eq. (21)

$$
\begin{aligned}
& F_{1}(x)=q_{1} x+\left(p_{1}-q_{1}\right) h(x) \\
& F_{2}(x)=q_{2} x+\left(p_{2}-q_{2}\right) h(x) \\
& F_{3}(x)=q_{3} x+\left(p_{3}-q_{3}\right) h(x)
\end{aligned}
$$




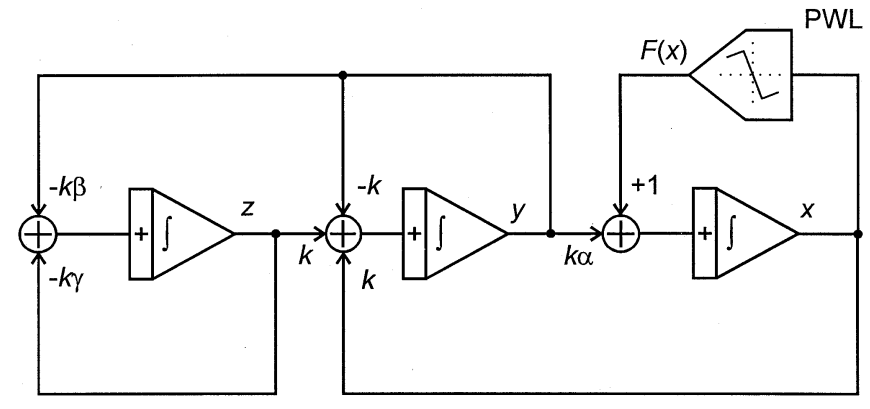

(a)

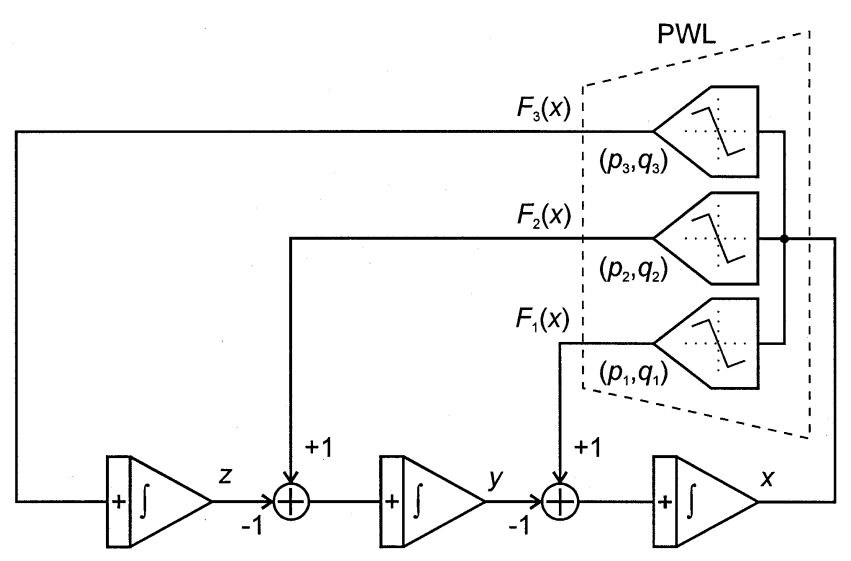

(b)

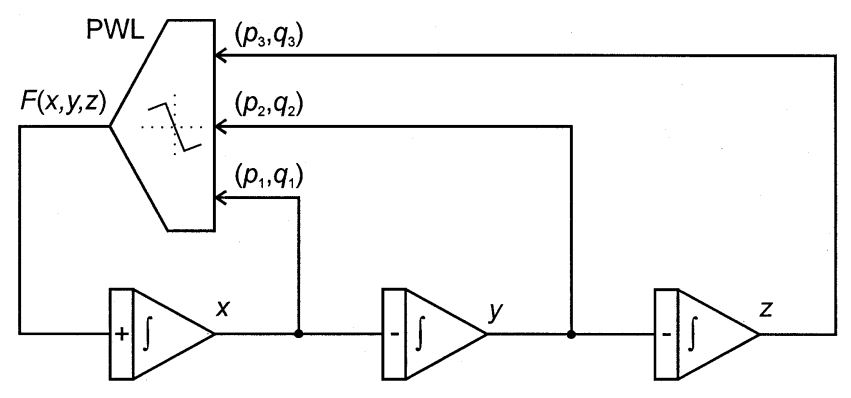

(c)

Fig. 5. Global forms of integrator-based block diagrams. (a) Chua's equations. (b) First canonical ODE equivalent. (c) Second canonical ODE equivalent.

the equation can be simplified to

$$
\begin{aligned}
& \dot{x}=-y+F_{1}(x) \\
& \dot{y}=-z+F_{2}(x) \\
& \dot{z}=F_{3}(x)
\end{aligned}
$$

It entails a very simple integrator-based block diagram with a single-input multiple-output PWL feedback block consisting of three subblocks, each of them determined by two equivalent eigenvalue parameters $p_{i}, q_{i}(i=1,2,3)$, as shown in Fig. 5(b).

\subsubsection{Second canonical ODE equivalent}

In this dual case Eq. (23) is rewritten into the simple global form

$$
\begin{aligned}
& \dot{x}=F(x, y, z) \\
& \dot{y}=-x \\
& \dot{z}=-y
\end{aligned}
$$

where

$$
\begin{aligned}
F(x, y, z)= & q_{1} x+q_{2} y+q_{3} z+h\left(\mathbf{w}^{\mathrm{T}} \mathbf{x}\right) \\
\mathbf{w}^{\mathrm{T}} \mathbf{x}= & \left(p_{1}-q_{1}\right) x+\left(p_{2}-q_{2}\right) y \\
& +\left(p_{3}-q_{3}\right) z
\end{aligned}
$$

The corresponding block diagram contains a multiple-input single-output PWL feedback block as shown in Fig. 5(c).

\subsection{Comparison of Chua's equations and their canonical $O D E$ equivalents}

All the three ODE forms considered can formally be compared from the following viewpoints:

\section{(i) Form of basic equations}

As follows from Eqs. (17), (22) and (24) the total number of nonzero elements in state matrix $\mathbf{A}$ and vectors $\mathbf{b}, \mathbf{w}$, remains the same in all three models. In Chua's equations both vectors $\mathbf{b}$ and $\mathbf{w}$ have the simple elementary form $\mathbf{b}=\mathbf{w}=\left(\begin{array}{lll}1 & 0 & 0\end{array}\right)^{\mathrm{T}}$, which indicates that the PWL function $h(\cdot)$ occurs only in the first equation and its argument is only given by variable $x$.

The first canonical ODE equivalent has the same elementary form of vector $\mathbf{w}$ as Chua's equations but the full form of vector $\mathbf{b}$ so that the PWL function $h(\cdot)$ appears in all the three equations. On the contrary, the second canonical ODE equivalent has the same elementary form of vector $\mathbf{b}$ but the full form of vector $\mathbf{w}$, and then the argument $\mathbf{w}^{\mathrm{T}} \mathbf{x}$ of function $h(\cdot)$ is given by the complete linear combination of all variables $x, y, z$.

\section{(ii) Design of model parameters}

For the design of Chua's equations it is necessary to calculate the set of dimensionless parameters $\{\alpha, \beta, \gamma, a, b, k\}$ from the chosen or given set of equivalent eigenvalue parameters 
$\left\{p_{1}, p_{2}, p_{3} ; q_{1}, q_{2}, q_{3}\right\}$ by Eqs. (19) and (20). These explicit formulae cannot be used in the cases where some denominators vanish, i.e. for the so-called set of measure zero $C_{0} \subset \Re^{6}$ [Chua, 1993]. In particular, any set of equivalent eigenvalue parameters satisfying at least one of the constraints

$$
p_{1}-q_{1}=0, \quad l_{1}=0, \quad l_{3}=0
$$

has an associated vector field belonging to $C_{0}$. However, such cases can be also solved with sufficient numerical accuracy by using the approximate model utilizing an arbitrarily small perturbation of any unrealizable eigenvalues [Chua et al., 1993a, 1993b].

In both canonical ODE equivalents the main model parameters are directly determined by the equivalent eigenvalue parameters so that they represent two exact dual models of Class $C$ including the set of measure zero. In fact, the requirement of such a universality was the main reason for the investigation of new canonical models [Pospíśil \& Brzobohatý, 1996a].

\section{(iii) Selection of bifurcation parameters}

Almost all the dimensionless parameters in Chua's equations, as well as the circuit parameters in Chua's oscillator, represent natural bifurcation parameters [Huang, 1996] so that using the changes of these individual parameters, several routes to chaos are simple to model [Chua et al., 1993a].

Since equivalent eigenvalue parameters $p_{i}$, $q_{i}(i=1,2,3)$ are not suitable for this purpose both canonical ODE equivalents cannot be used for a simple modeling of bifurcation phenomena in systems belonging to Class $C$. If this is required, simultaneous changes in several equivalent eigenvalue parameters are necessary.

\section{(iv) Realization of circuit models}

Chua's oscillator, as the simple circuit model corresponding to Chua's equations, represents the circuit structure convenient for practical realizations in electronic form. It contains only one nonlinear resistor [Fig. 1(c)] realizable as a standard IC electronic device and can be used in many conventional and unconventional applications including synchronized systems. Other types of circuit model derived directly from Eq. (16) can also be realized by using either CNN or integrator-based structures [Fig. 3(a)].

Simple forms of both canonical ODE equivalents given by Eqs. (21) and (23) are suitable for direct modeling by using the integratorbased circuit structures with one nonlinear feedback block only [Figs. 3(b), 3(c), 4(b) and 4(c)]. For several reasons the first form seems to be more convenient as a prototype for practical circuit realization either as the analog system [Hanus, 1997] or with digital setting of parameters [Kolka \& Michálek, 1996; Hanus, 1998], as well as for the CNN technique [Kaderka, 1996; Švajda \& Kaderka, 1998].

For the realization of all circuit models the sensitivity properties of various prototypes are very important. Especially the eigenvalue sensitivities with respect to the individual parameter changes can play a significant role and they represent the subject of further useful comparative study in this field [Horská, 1999].

\section{(v) Dynamical behavior}

To demonstrate graphically the similar dynamical behavior of all the ODE forms considered, several 3-D phase-portraits for different sets of equivalent eigenvalue parameters $p_{i}, q_{i}(i=1,2,3)$ are shown in Figs. 6-9. This example represents the period-doubling route to chaos [Chua et al., 1993a] where the individual models are distinguished by different colors of the trajectories, by subscripts of the individual variables, and by scales on the axes, i.e.

(a) Chua's model: red color

$\mathbf{x} \triangleq \mathbf{x}_{\mathrm{C}}=\left(\begin{array}{lll}x_{\mathrm{C}} & y_{\mathrm{C}} \quad z_{\mathrm{C}}\end{array}\right)^{\mathrm{T}}, \quad x_{\mathrm{C}}=-5 \quad$ to $5, \quad y_{\mathrm{C}}=-0.5 \quad$ to $0.5, \quad z_{\mathrm{C}}=-4$ to $4 ;$

(b) First canonical form: blue color

$\mathbf{x} \triangleq \mathbf{x}_{\mathrm{I}}=\left(\begin{array}{lll}x_{\mathrm{I}} & y_{\mathrm{I}} & z_{\mathrm{I}}\end{array}\right)^{\mathrm{T}}, \quad x_{\mathrm{I}}=-5$ to $5, \quad y_{\mathrm{I}}=-5$ to $5, \quad z_{\mathrm{I}}=-20$ to $20 ;$

(c) Second canonical form: green color

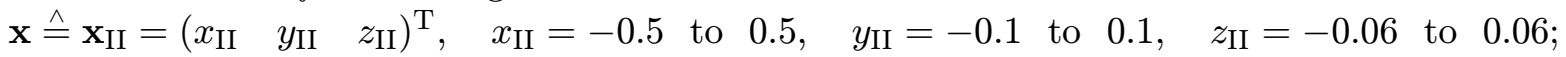

Note that such a sequence of trajectories can be obtained from Chua's equations by changing only parameter $\alpha$, i.e. only parameter $C_{1}$ in Chua's oscillator. (In Figs. 6-8 the trajectories are separated into two symmetric parts according to different initial conditions.) 

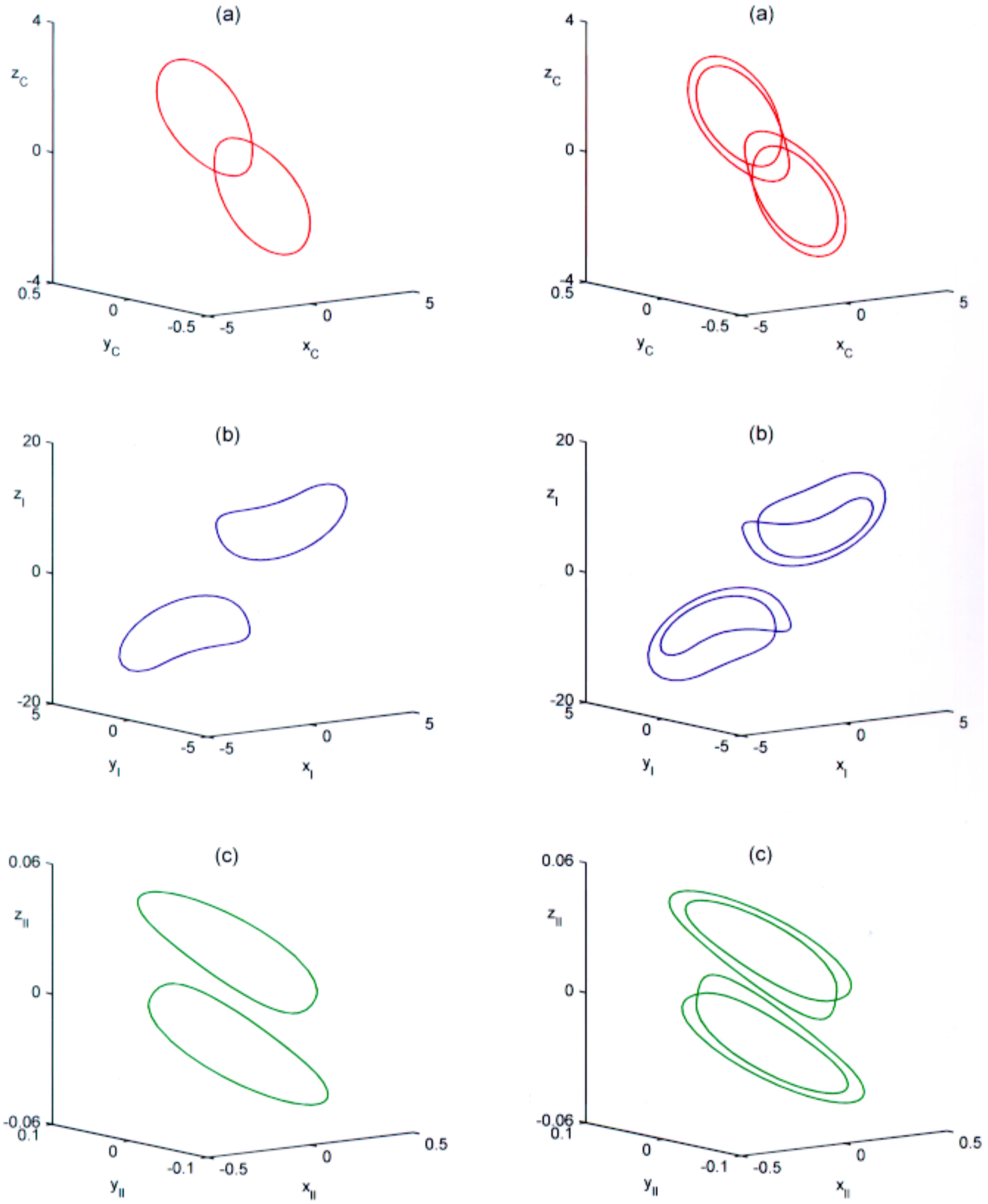

Fig. 6. Doubled period route to chaos. Step 1: Two symmetric period-1 orbits. (a) Chua's equations. (b) First canonical ODE equivalent. (c) Second canonical ODE equivalent. $\left(p_{1}=0.25835 ; p_{2}=5.94192 ; p_{3}=20.13375 ; q_{1}=-3.51671 ;\right.$ $q_{2}=9.717 ; q_{3}=-40.26751$.)

Fig. 7. Doubled period route to chaos. Step 2: Two symmetric period-2 orbits. (a) Chua's equations. (b) First canonical ODE equivalent. (c) Second canonical ODE equivalent. $\left(p_{1}=0.29411 ; p_{2}=5.65610 ; p_{3}=20.70588 ; q_{1}=-3.58823 ;\right.$ $\left.q_{2}=9.53846 ; q_{3}=-41.41176.\right)$ 

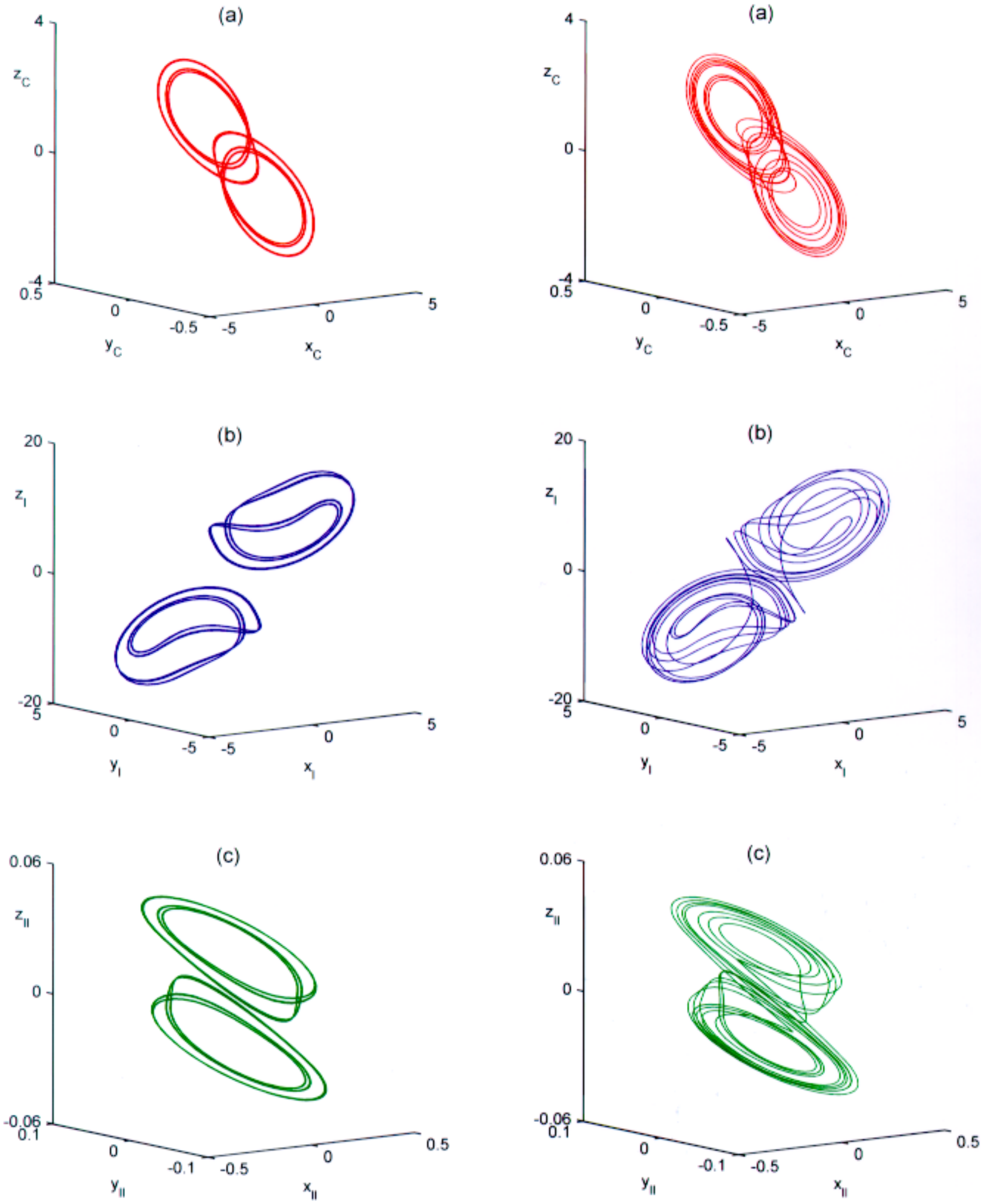

Fig. 8. Doubled period route to chaos. Step 3: Two symmetric period-4 orbits (a) Chua's equations. (b) First canonical ODE equivalent. (c) Second canonical ODE equivalent. $\left(p_{1}=0.30593 ; p_{2}=5.56164 ; p_{3}=20.89497 ; q_{1}=-3.61187\right.$; $q_{2}=9.47945 ; q_{3}=-41.78995$.)

Fig. 9. Doubled period route to chaos. Step 4: Symmetric trajectories of chaotic attractor. (a) Chua's equations. (b) First canonical ODE equivalent. (c) Second canonical ODE equivalent. $\left(p_{1}=0.40141 ; p_{2}=4.79851 ; p_{3}=\right.$ $\left.22.42257 ; q_{1}=-3.80282 ; q_{2}=9.00274 ; q_{3}=-44.84515.\right)$ 
Exact mutual relations among all the three ODE forms expressed by the linear topological conjugacy are given in Sec. 3, where not only variables but also all matrices and vectors corresponding to the individual models are designated by different subscripts, i.e.

(a) Chua's model - Eqs. (17a)-(17b): $\mathbf{A} \triangleq \mathbf{A}_{\mathrm{C}}, \quad \mathbf{b} \triangleq \mathbf{b}_{\mathrm{C}}, \quad \mathbf{w} \triangleq \mathbf{w}_{\mathrm{C}}$;

(b) First canonical form - Eq. (22): $\mathbf{A} \triangleq \mathbf{A}_{\mathrm{I}}, \quad \mathbf{b} \triangleq \mathbf{b}_{\mathrm{I}}, \quad \mathbf{w} \triangleq \mathbf{w}_{\mathrm{I}}$;

(c) Second canonical form - Eq. (24): $\mathbf{A} \triangleq \mathbf{A}_{\mathrm{II}}, \quad \mathbf{b} \triangleq \mathbf{b}_{\mathrm{II}}, \quad \mathbf{w} \triangleq \mathbf{w}_{\mathrm{II}}$;

\section{Qualitative Equivalence of Individual ODE Forms}

To express mutual relations among all the three ODE equivalents considered, the conditions of linear topological conjugacy are first defined. Then they are applied to individual ODE forms and the "similarity" transformations between the two of them are derived. Finally, the graphical illustration of this transformation is shown using the socalled "elastic space" analogy demonstrating the gradual conversion of one ODE form trajectory into another.

\subsection{Linear topological conjugacy}

Definition. Any two systems of Class $C$ having the same values of the two sets of eigenvalues $\left\{\mu_{1}, \mu_{2}, \mu_{3} ; \nu_{1}, \nu_{2}, \nu_{3}\right\}$ or equivalent eigenvalue parameters $\left\{p_{1}, p_{2}, p_{3} ; q_{1} ; q_{2} ; q_{3}\right\}$ are qualitatively equivalent in their dynamical behavior. Mutual relations between these systems can be expressed by a linear topological conjugacy in accordance with the global unfolding theorem [Chua, 1993] and the global properties of linear ordinary differential equations [Neuman, 1991].

In the general case, two autonomous PWL dynamical systems belonging to Class $C$ described by the general matrix form given by Eq. (7) can be considered, i.e.

$$
\dot{\mathbf{x}}=\mathbf{A} \mathbf{x}+\mathbf{b} h\left(\mathbf{w}^{\mathrm{T}} \mathbf{x}\right)
$$

and

$$
\dot{\tilde{\mathbf{x}}}=\tilde{\mathbf{A}} \tilde{\mathbf{x}}+\tilde{\mathbf{b}} h\left(\tilde{\mathbf{w}}^{\mathrm{T}} \tilde{\mathbf{x}}\right) .
$$

If these two systems are linearly conjugate (i.e. topologically conjugate through a linear map), the linear relation between the two sets of variables $\mathbf{x}$ and $\tilde{\mathbf{x}}$ can be expressed explicitly as

$$
\mathbf{x}=\mathbf{T} \tilde{\mathbf{x}}
$$

or in the inverse form

$$
\tilde{\mathbf{x}}=\tilde{\mathbf{T}} \mathbf{x}, \quad \tilde{\mathbf{T}}=\mathbf{T}^{-1}
$$

where $\mathbf{T}$ is a nonsingular transformation matrix. Substituting Eq. (36a) into Eq. (35a) and rewriting it into the form of Eq. (35b) the formal relations between state matrices $\mathbf{A}$ and $\tilde{\mathbf{A}}$ as well as between vectors $\mathbf{b}, \mathbf{w}$ and $\tilde{\mathbf{b}}, \tilde{\mathbf{w}}$ of both the systems can explicitly be expressed as

$$
\begin{aligned}
\mathbf{A} & =\mathbf{T} \tilde{\mathbf{A}} \mathbf{T}^{-1} \\
\mathbf{b} & =\mathbf{T} \tilde{\mathbf{b}} \\
\mathbf{w}^{\mathrm{T}} & =\tilde{\mathbf{w}}^{\mathrm{T}} \mathbf{T}
\end{aligned}
$$

or in the inverse form

$$
\begin{gathered}
\tilde{\mathbf{A}}=\mathbf{T}^{-1} \mathbf{A} \mathbf{T}=\tilde{\mathbf{T}} \mathbf{A} \tilde{\mathbf{T}}^{-1} \\
\tilde{\mathbf{b}}=\mathbf{T}^{-1} \mathbf{b}=\tilde{\mathbf{T}} \mathbf{b} \\
\tilde{\mathbf{w}}^{\mathrm{T}}=\mathbf{w}^{\mathrm{T}} \mathbf{T}^{-1}=\mathbf{w}^{\mathrm{T}} \tilde{\mathbf{T}}
\end{gathered}
$$

Transformation matrices $\mathbf{T}$ and $\tilde{\mathbf{T}}$ can be considered as products, i.e.

$$
\mathbf{T}=\mathbf{K}^{-1} \tilde{\mathbf{K}}, \quad \tilde{\mathbf{T}}=\tilde{\mathbf{K}}^{-1} \mathbf{K}=\mathbf{T}^{-1}
$$

where $\mathbf{K}$ and $\tilde{\mathbf{K}}$ represent partial nonsingular transformation matrices of the first and the second systems, respectively. Then the explicit relations given by Eqs. (36)-(38) can be rewritten into the implicit form

$$
\begin{aligned}
\mathbf{K} \mathbf{x} & =\tilde{\mathbf{K}} \tilde{\mathbf{x}} \\
\mathbf{K} \mathbf{A} \mathbf{K}^{-1} & =\tilde{\mathbf{K}} \tilde{\mathbf{A}} \tilde{\mathbf{K}}^{-1} \\
\mathbf{K} \mathbf{b} & =\tilde{\mathbf{K}} \tilde{\mathbf{b}} .
\end{aligned}
$$

Partial transformation matrices $\mathbf{K}$ and $\tilde{\mathbf{K}}$ are generally given by nonsingular forms

$$
\mathbf{K}=\mathbf{K}\left(\mathbf{w}^{\mathrm{T}}, \mathbf{A}\right)=\left[\begin{array}{c}
\mathbf{w}^{\mathrm{T}} \\
\mathbf{w}^{\mathrm{T}} \mathbf{A} \\
\mathbf{w}^{\mathrm{T}} \mathbf{A}^{2}
\end{array}\right]
$$


and

$$
\tilde{\mathbf{K}}=\tilde{\mathbf{K}}\left(\tilde{\mathbf{w}}^{\mathrm{T}}, \tilde{\mathbf{A}}\right)=\left[\begin{array}{c}
\tilde{\mathbf{w}}^{\mathrm{T}} \\
\tilde{\mathbf{w}}^{\mathrm{T}} \tilde{\mathbf{A}} \\
\tilde{\mathbf{w}}^{\mathrm{T}} \tilde{\mathbf{A}}^{2}
\end{array}\right]
$$

fulfilling the observability conditions of pairs $\left(\mathbf{w}^{\mathrm{T}}, \mathbf{A}\right)$ and $\left(\tilde{\mathbf{w}}^{\mathrm{T}}, \tilde{\mathbf{A}}\right)$, respectively. A rigorous proof, including all details extended for the Lur'e systems, is introduced in [Wu \& Chua, 1996]. Here, only a simplified procedure is given that verifies formally the validity of Eqs. (39)-(41), where matrices $\mathbf{K}$ are $\tilde{\mathbf{K}}$ expressed by Eqs. (42a)-(42b).

As each inner expression in matrices $\mathbf{K}$ and $\tilde{\mathbf{K}}$ represents a complete matrix row, the basic global implicit relations given by Eqs. (39) and (41) can be rewritten into the following two sets of three partial conditions, i.e. for variables $\mathbf{x}$ and $\tilde{\mathbf{x}}$ as

$$
\begin{aligned}
\mathbf{w}^{\mathrm{T}} \mathbf{x} & =\tilde{\mathbf{w}}^{\mathrm{T}} \tilde{\mathbf{x}} \\
\mathbf{w}^{\mathrm{T}} \mathbf{A} \mathbf{x} & =\tilde{\mathbf{w}}^{\mathrm{T}} \tilde{\mathbf{A}} \tilde{\mathbf{x}} \\
\mathbf{w}^{\mathrm{T}} \mathbf{A}^{2} \mathbf{x} & =\tilde{\mathbf{w}}^{\mathrm{T}} \tilde{\mathbf{A}}{ }^{2} \tilde{\mathbf{x}}
\end{aligned}
$$

and for vectors $\mathbf{b}$ and $\tilde{\mathbf{b}}$ as

$$
\begin{aligned}
\mathbf{w}^{\mathrm{T}} \mathbf{b} & =\tilde{\mathbf{w}}^{\mathrm{T}} \tilde{\mathbf{b}} \\
\mathbf{w}^{\mathrm{T}} \mathbf{A} \mathbf{b} & =\tilde{\mathbf{w}}^{\mathrm{T}} \tilde{\mathbf{A}} \tilde{\mathbf{b}} \\
\mathbf{w}^{\mathrm{T}} \mathbf{A}^{2} \mathbf{b} & =\tilde{\mathbf{w}}^{\mathrm{T}} \tilde{\mathbf{A}}^{2} \tilde{\mathbf{b}} .
\end{aligned}
$$

The partial conditions given by Eq. (39a) follow directly from the basic requirement for the equality of the arguments of the PWL function $h(\cdot)$ in Eqs. (35a) and (35b). The substitution of $\dot{\mathbf{x}}$ and $\dot{\tilde{\mathbf{x}}}$ from these basic equations into the first derivative of Eq. (39a) gives

$$
\mathbf{w}^{\mathrm{T}}\left[\mathbf{A} \mathbf{x}+\mathbf{b} h\left(\mathbf{w}^{\mathrm{T}} \mathbf{x}\right)\right]=\tilde{\mathbf{w}}^{\mathrm{T}}\left[\tilde{\mathbf{A}} \tilde{\mathbf{x}}+\tilde{\mathbf{b}} h\left(\tilde{\mathbf{w}}^{\mathrm{T}} \tilde{\mathbf{x}}\right)\right]
$$

It is evident that the partial conditions given by Eqs. (39a) and (41a) follow directly from the equality of the linear and the PWL parts in Eq. (43), respectively. Similarly, using the first derivative of Eq. (39b) and substituting from Eqs. (35a) and (35b), the following expression is obtained

$$
\mathbf{w}^{\mathrm{T}}\left[\mathbf{A}^{2} \mathbf{x}+\mathbf{A} \mathbf{b} h\left(\mathbf{w}^{\mathrm{T}} \mathbf{x}\right)\right]=\tilde{\mathbf{w}}^{\mathrm{T}}\left[\tilde{\mathbf{A}}^{2} \tilde{\mathbf{x}}+\tilde{\mathbf{A}} \tilde{\mathbf{b}} h\left(\tilde{\mathbf{w}}^{\mathrm{T}} \tilde{\mathbf{x}}\right)\right] .
$$

Owing to the above equality of the linear and PWL parts, the partial conditions given by Eqs. (39c) and (41b) can be obtained. Finally, using the same comparative procedure and starting from the first derivative of the basic condition given by Eq. (39), the related state matrix transformation given by Eq. (40) and the complete vector transformation given by Eq. (41) can be easily derived. In this way the last partial condition given by Eq. (41c) is also automatically proved because it represents the third row of Eq. (41).

\subsection{Mutual relations of individual $O D E$ forms}

The first partial transformation matrices associated with the individual models are derived from Eq. (42). In accordance with Sec. 2.5 they are also distinguished by different subscripts, i.e. $\mathbf{K}_{\mathrm{C}}, \mathbf{K}_{\mathrm{I}}$, $\mathbf{K}_{\mathrm{II}}$.

\subsubsection{Chua's model}

Substituting state matrix $\mathbf{A} \triangleq \mathbf{A}_{\mathrm{C}}$ from Eq. (17a) and vector $\mathbf{w} \triangleq \mathbf{w}_{\mathrm{C}}$ from Eq. (17b) into Eq. (42) the corresponding partial transformation matrix is obtained in the lower-triangular form

$$
\mathbf{K}_{\mathrm{C}}=\left[\begin{array}{ccc}
1 & 0 & 0 \\
-k \alpha(b+1) & k \alpha & 0 \\
\alpha\left[1+\alpha(b+1)^{2}\right] & -\alpha[1+\alpha(b+1)] & \alpha
\end{array}\right]
$$

\subsubsection{First canonical form}

Starting from state matrix $\mathbf{A} \triangleq \mathbf{A}_{\mathrm{I}}$ and vector $\mathbf{w} \hat{=} \mathbf{w}_{\text {I }}$ from Eq. (22) the corresponding partial transformation matrix is obtained from Eq. (42) also in the lower-triangular form

$$
\mathbf{K}_{\mathrm{I}}=\left[\begin{array}{ccc}
1 & 0 & 0 \\
q_{1} & -1 & 0 \\
q_{1}^{2}-q_{2} & -q_{1} & 1
\end{array}\right]
$$

The first canonical form is very convenient as a simple and universal reference model because the inverse form of its partial transformation matrix is also very simple, i.e.

$$
\mathbf{K}_{\mathrm{I}}^{-1}=\left[\begin{array}{ccc}
1 & 0 & 0 \\
q_{1} & -1 & 0 \\
q_{2} & -q_{1} & 1
\end{array}\right]
$$

\subsubsection{Second canonical form}

Utilizing state matrix $\mathbf{A} \bumpeq \mathbf{A}_{\mathrm{II}}$ and vector $\mathbf{w} \hat{=} \mathbf{w}_{\mathrm{II}}$ from Eq. (24) the corresponding partial transformation matrix obtained from Eq. (42) can be expressed as 


$$
\mathbf{K}_{\mathrm{II}}=\left[\begin{array}{ccc}
p_{1}-q_{1} & p_{2}-q_{2} & p_{3}-q_{3} \\
q_{1}\left(p_{1}-q_{1}\right)-\left(p_{2}-q_{2}\right) & q_{2}\left(p_{1}-q_{1}\right)-\left(p_{3}-q_{3}\right) & q_{3}\left(p_{1}-q_{1}\right) \\
K_{31} & K_{32} & K_{33}
\end{array}\right]
$$

where

$$
\begin{aligned}
& K_{31}=\left(q_{1}^{2}-q_{2}\right)\left(p_{1}-q_{1}\right)-q_{1}\left(p_{2}-q_{2}\right)-\left(p_{3}-q_{3}\right) \\
& K_{32}=\left(q_{1} q_{2}-q_{3}\right)\left(p_{1}-q_{1}\right)-q_{2}\left(p_{2}-q_{2}\right) \\
& K_{33}=q_{3}\left[q_{1}\left(p_{1}-q_{1}\right)-\left(p_{2}-q_{2}\right)\right]
\end{aligned}
$$

Now the complete nonsingular transformation matrix $\mathbf{T}$ can be calculated from Eq. (38) for any two ODE forms considered and the explicit relations between the corresponding variables can be expressed in accordance with Eq. (36a).

(i) Chua's model $\Leftrightarrow$ First canonical form

Denoting $\mathbf{x} \triangleq \mathbf{x}_{\mathrm{I}}, \mathbf{K} \triangleq \mathbf{K}_{\mathrm{I}}, \tilde{\mathbf{x}} \triangleq \mathbf{x}_{\mathrm{C}}, \tilde{\mathbf{K}} \triangleq \mathbf{K}_{\mathrm{C}}$, and
$\mathbf{T} \triangleq \mathbf{T}_{\mathrm{C}-\mathrm{I}}$, Eqs. $(36 \mathrm{a})-(38)$ can be written as

$$
\begin{aligned}
\mathbf{x}_{\mathrm{I}} & =\mathbf{T}_{\mathrm{C}-\mathrm{I}} \mathbf{x}_{\mathrm{C}} \\
\mathbf{b}_{\mathrm{I}} & =\mathbf{T}_{\mathrm{C}-\mathrm{I}} \mathbf{b}_{\mathrm{C}} \\
\mathbf{A}_{\mathrm{I}} & =\mathbf{T}_{\mathrm{C}-\mathrm{I}} \mathbf{A}_{\mathrm{C}} \mathbf{T}_{\mathrm{C}-\mathrm{I}}^{-1}
\end{aligned}
$$

where

$$
\mathbf{T}_{\mathrm{C}-\mathrm{I}}=K_{\mathrm{I}}^{-1} \mathbf{K}_{\mathrm{C}} .
$$

Here the double subscript of the complete transformation matrix indicates that the qualitatively equivalent first canonical form is expressed by Chua's model. Substituting the partial transformation matrices from Eqs. (45) and (46) into Eq. (49d) the corresponding resultant transformation matrix is

$$
\mathbf{T}_{\mathrm{C}-\mathrm{I}}=\left[\begin{array}{ccc}
1 & 0 & 0 \\
q_{1}+k \alpha(b+1) & -k \alpha & 0 \\
q_{2}+q_{1} k \alpha(b+1)+\alpha\left[1+\alpha(b+1)^{2}\right] & -q_{1} k \alpha-\alpha[1+\alpha(b+1)] & \alpha
\end{array}\right]
$$

Note that parameters $\alpha$ and $b$ depend on equivalent eigenvalue parameters $p_{i}, q_{i}(i=1,2,3)$ according to Eq. (19) so that the full form of matrix $\mathbf{T}_{\mathrm{C}-\mathrm{I}}$ is more complex.

\section{(ii) Second canonical form $\Leftrightarrow$ First canonical form}

In this case $\mathbf{x} \triangleq \mathbf{x}_{\mathrm{I}}, \mathbf{K} \triangleq \mathbf{K}_{\mathrm{I}}, \tilde{\mathbf{x}} \triangleq \mathbf{x}_{\mathrm{II}}, \tilde{\mathbf{K}} \triangleq \mathbf{K}_{\mathrm{II}}$, $\mathbf{T} \triangleq \mathbf{T}_{\text {II-I }}$, and Eqs. (36a)-(38) can be rewritten as

$$
\begin{aligned}
& \mathbf{x}_{\mathrm{I}}=\mathbf{T}_{\mathrm{II}-\mathrm{I}} \mathbf{x}_{\mathrm{II}} \\
& \mathbf{b}_{\mathrm{I}}=\mathbf{T}_{\mathrm{II}-\mathrm{I}} \mathbf{b}_{\mathrm{II}}
\end{aligned}
$$

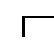

$$
\mathbf{A}_{\mathrm{I}}=\mathbf{T}_{\mathrm{II}-\mathrm{I}} \mathbf{A}_{\mathrm{II}} \mathbf{T}_{\mathrm{II}-\mathrm{I}}^{-1}
$$

where

$$
\mathbf{T}_{\mathrm{II}-\mathrm{I}}=\mathbf{K}_{\mathrm{I}}^{-1} \mathbf{K}_{\mathrm{II}}
$$

The double subscript of the complete transformation matrix here indicates that the qualitatively equivalent first canonical form is expressed by the second canonical form. Substituting the partial transformation matrices from Eqs. (46)-(48) into Eq. (51d) the corresponding resultant transformation matrix is directly obtained in its full form

$$
\mathbf{T}_{\mathrm{II}-\mathrm{I}}=\left[\begin{array}{ccc}
p_{1}-q_{1} & p_{2}-q_{2} & p_{3}-q_{3} \\
p_{2}-q_{2} & q_{1} p_{2}-q_{2} p_{1}+p_{3}-q_{3} & q_{1} p_{3}-q_{3} p_{1} \\
p_{3}-q_{3} & q_{1} p_{3}-q_{3} p_{1} & q_{2} p_{3}-q_{3} p_{2}
\end{array}\right]
$$


1-st canonical (a)

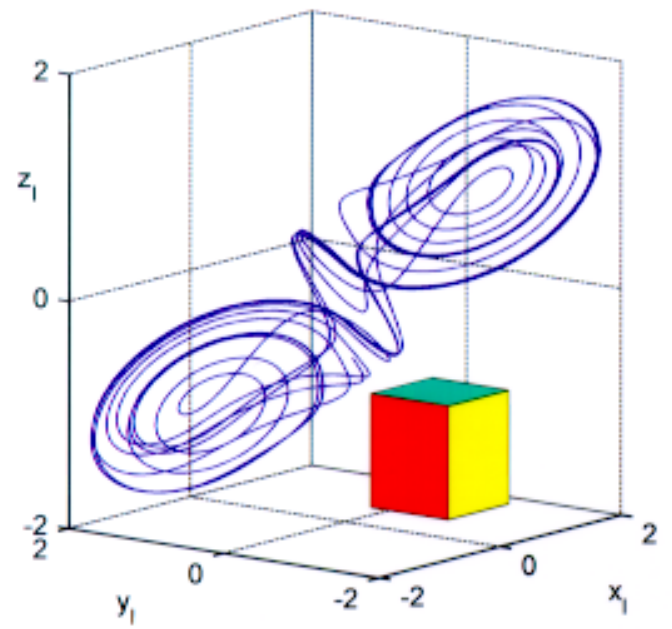

(c)

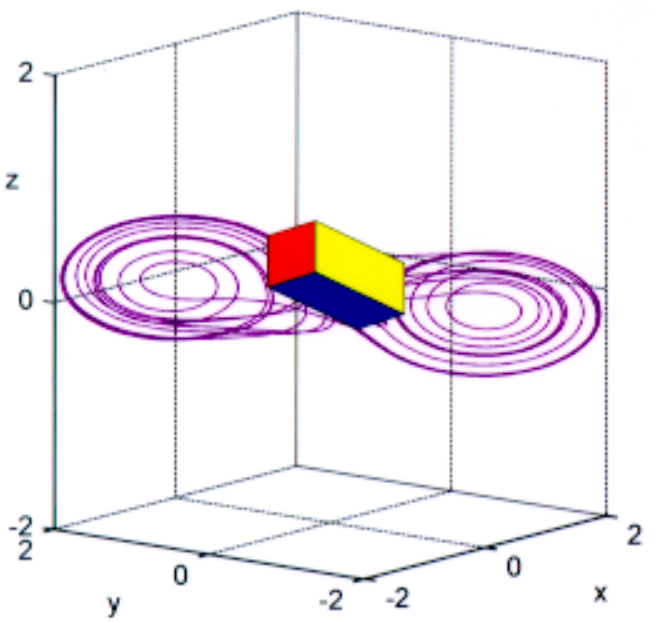

(e)

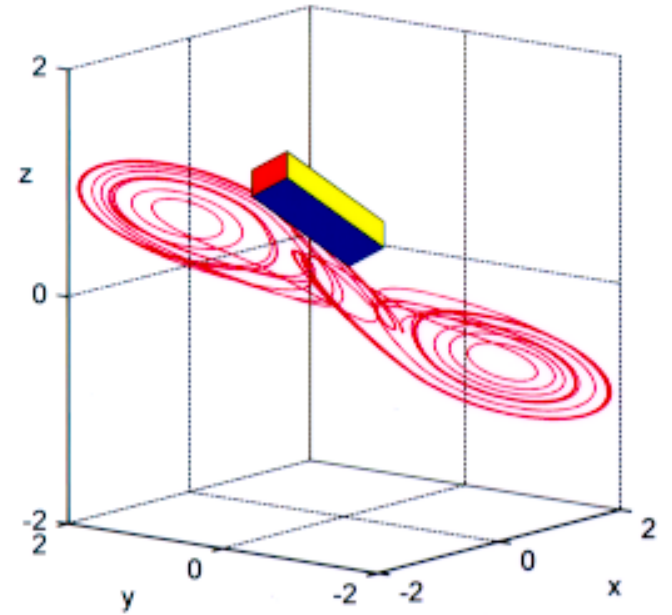

(b)

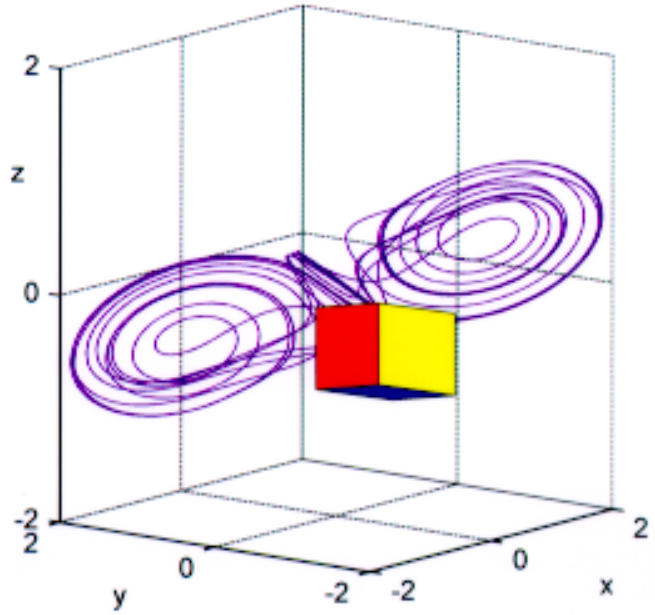

(d)

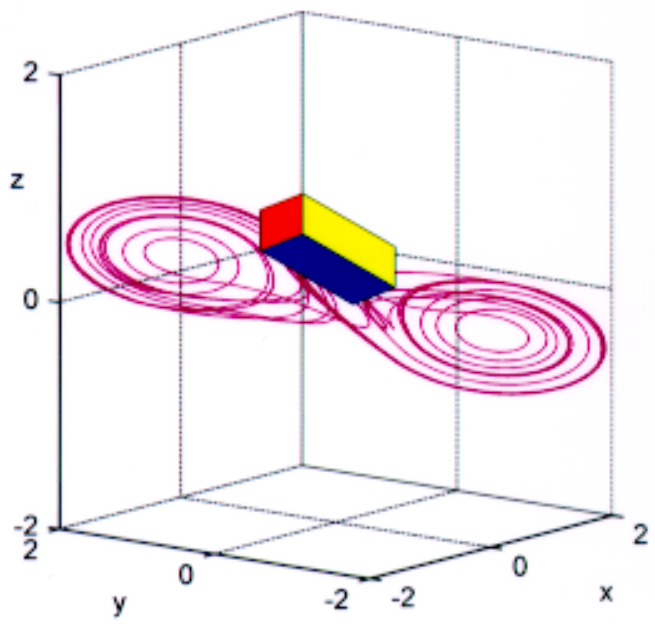

Chua's model (f)

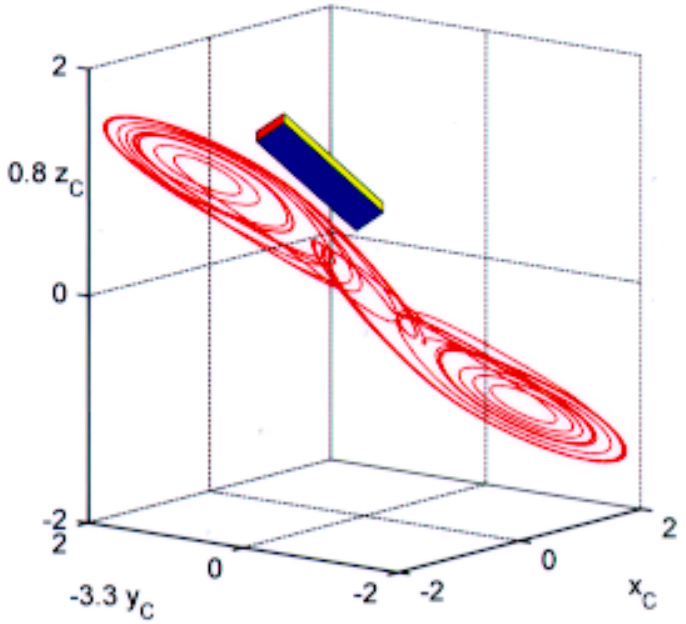

Fig. 10. Graphical illustration of linear topological conjugacy using "elastic space" analogy. (a) First canonical form. (b)-(e) Transient step. (f) Chua's model. $\quad\left(p_{1}=0.09 ; p_{2}=0.432961 ; p_{3}=0.653325 ; q_{1}=-1.168 ; q_{2}=0.846341\right.$; $q_{3}=-1.2948$.) 
1-st canonical (a)

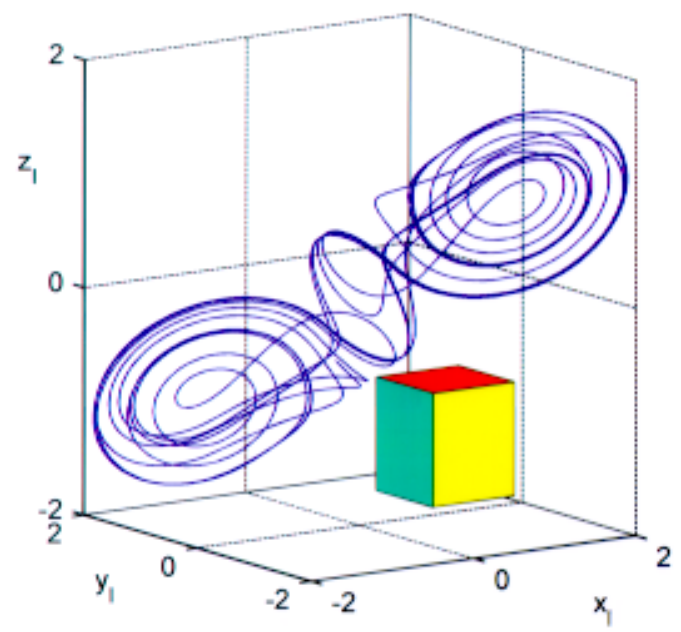

(c)

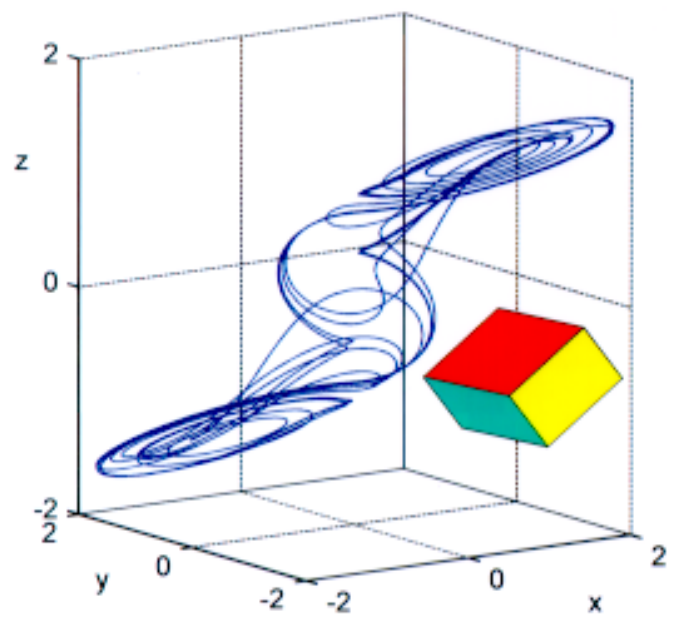

(e)

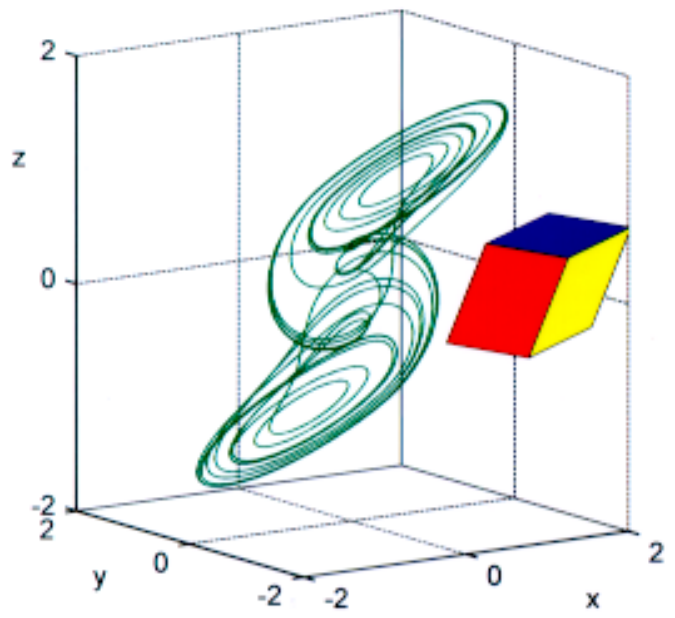

(b)

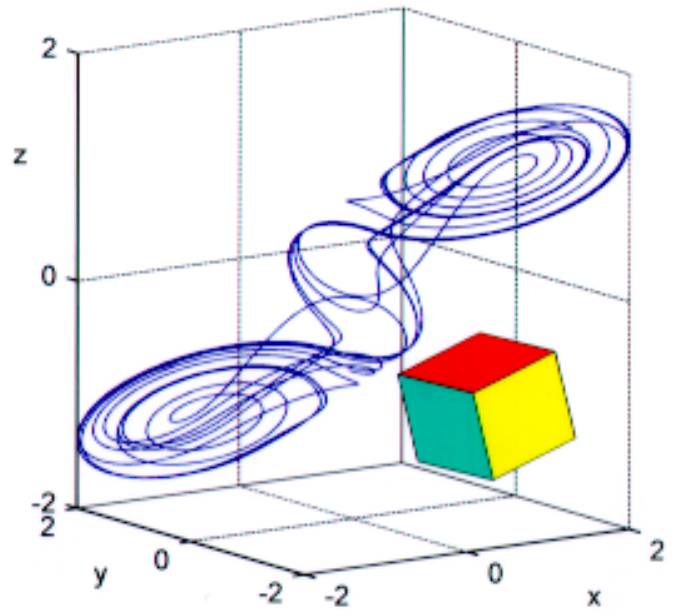

(d)

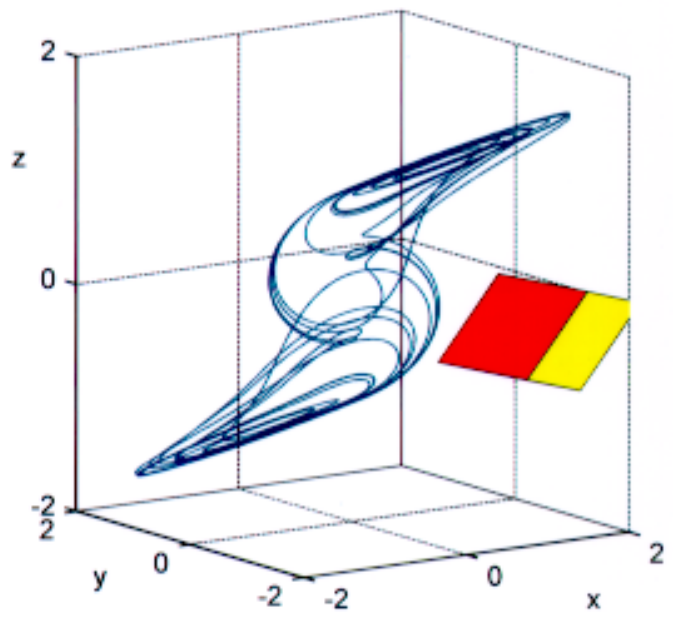

2-nd canonical (f)

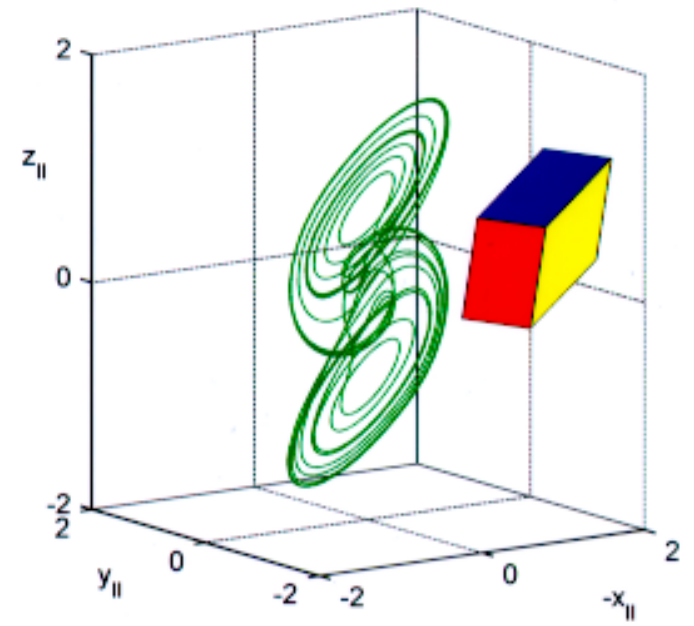

Fig. 11. Graphical illustration of linear topological conjugacy using "elastic space" analogy. (a) First canonical form. (b)-(e) Transient steps. (f) Second canonical form. ( $p_{1}=0.09 ; p_{2}=0.432961 ; p_{3}=0.653325 ; q_{1}=-1.168 ; q_{2}=0.846341$; $q_{3}=-1.2948$.) 
The symmetry of this matrix follows from the mutual duality of both canonical ODE forms.

\section{(iii) Chua's model $\Leftrightarrow$ Second canonical form}

Here $\mathbf{x} \triangleq \mathbf{x}_{\mathrm{II}}, \mathbf{K} \triangleq \mathbf{K}_{\mathrm{II}}, \tilde{\mathbf{x}} \triangleq \mathbf{x}_{\mathrm{C}}, \tilde{\mathbf{K}} \triangleq \mathbf{K}_{\mathrm{C}}$, and $\mathbf{T} \triangleq \mathbf{T}_{\mathrm{C}-\mathrm{II}}$, and Eqs. (36a)-(38) can be rewritten into the forms

$$
\begin{aligned}
\mathbf{x}_{\mathrm{II}} & =\mathbf{T}_{\mathrm{C}-\mathrm{II}} \mathbf{x}_{\mathrm{C}} \\
\mathbf{b}_{\mathrm{II}} & =\mathbf{T}_{\mathrm{C}-\mathrm{II}} \mathbf{b}_{\mathrm{C}} \\
\mathbf{A}_{\mathrm{II}} & =\mathbf{T}_{\mathrm{C}-\mathrm{II}} \mathbf{A}_{\mathrm{C}} \mathbf{T}_{\mathrm{C}-\mathrm{II}}^{-1}
\end{aligned}
$$

where

$$
\mathbf{T}_{\mathrm{C}-\mathrm{II}}=\mathbf{K}_{\mathrm{II}}^{-1} \mathbf{K}_{\mathrm{C}}
$$

This form corresponds to the fact that the second canonical form is expressed by Chua's model. Substituting the partial transformation matrices from Eqs. (45)-(48) into Eq. (53d) the corresponding complete transformation matrix can be obtained. Its full symbolic form is more complex and not given here. The same transformation can be evidently expressed by a combination of the two previous cases, where the resultant transformation matrix is given as the product

$$
\mathbf{T}_{\mathrm{C}-\mathrm{II}}=\mathbf{K}_{\mathrm{II}}^{-1} \mathbf{K}_{\mathrm{I}} \mathbf{K}_{\mathrm{I}}^{-1} \mathbf{K}_{\mathrm{C}}=\mathbf{T}_{\mathrm{II}-\mathrm{I}}^{-1} \mathbf{T}_{\mathrm{C}-\mathrm{I}}
$$

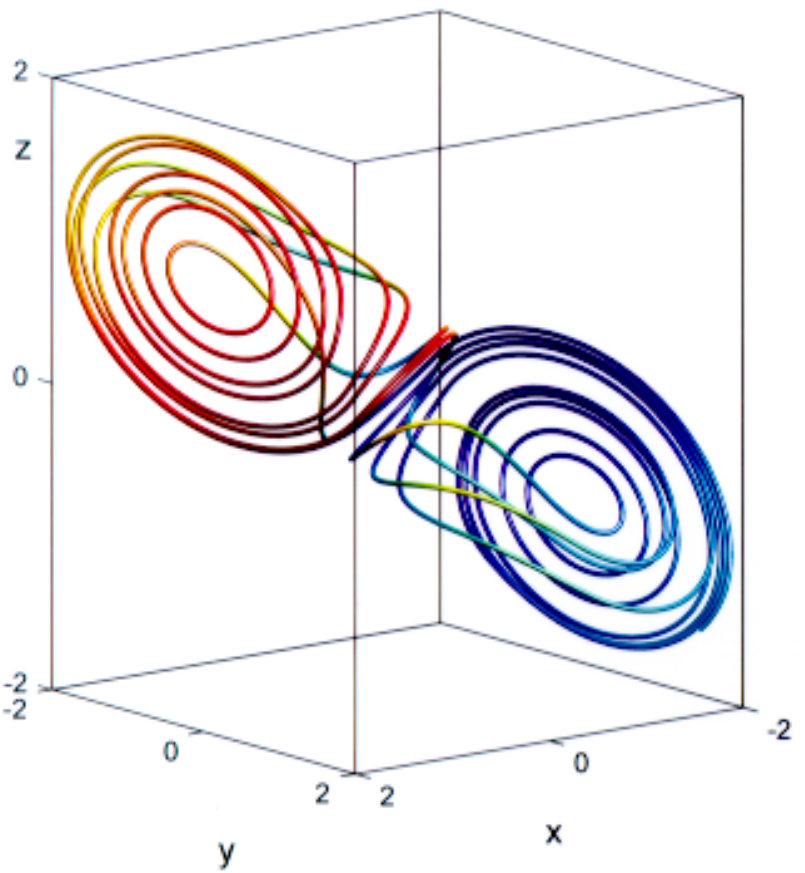

\subsection{Graphical illustration}

The linear topological conjugacy among individual ODE forms is graphically illustrated in Figs. 10 and 11 by the phase-portraits of the double scroll attractor utilizing the procedure known as the "elastic space" analogy. In this way, the main geometrical aspects of similarity transformation are demonstrated by the sequence of trajectories starting in both cases from the first canonical form and continuously changing either to Chua's model (Fig. 10) or to the second canonical form (Fig. 11). It corresponds to the resultant transformation matrix in linear topological conjugacy condition given by Eq. (49a) or Eq. (51a). This step-by-step procedure is visualized by changing the color of the trajectories where the individual transient steps correspond to a certain hue of two basic colors in accordance with those used in Figs. 6-9. The small colored cubes in Figs. 10-11 indicate the "elastic space" deformation (rotation, contraction, dilatation, and angle change) during this gradual conversion. The convenient size of the final trajectories in Fig. 10(f) (Chua's model) is achieved by scaling the $y_{\mathrm{C}}$ and $z_{\mathrm{C}}$ coordinates. The negative value of the corresponding coefficient in the $y_{\mathrm{C}}$ axis represents the additional trajectory reflection around the $x_{\mathrm{C}}-z_{\mathrm{C}}$ plane similarly to the negative sign of the $x_{\text {II }}$ in

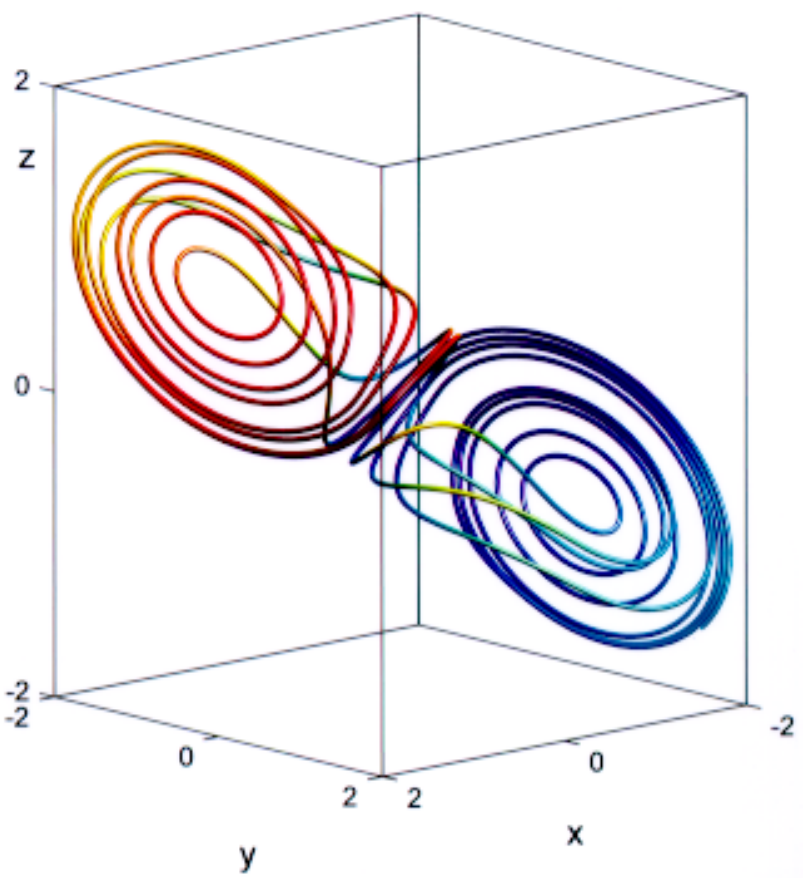

Fig. 12. Stereoscopic view to 3 -D phase-portrait of Chua's double-scroll chaotic attractor. $\quad\left(p_{1}=0.09 ; p_{2}=0.432961\right.$; $p_{3}=0.653325 ; q_{1}=-1.168 ; q_{2}=0.846341 ; q_{3}=-1.2948$.) 

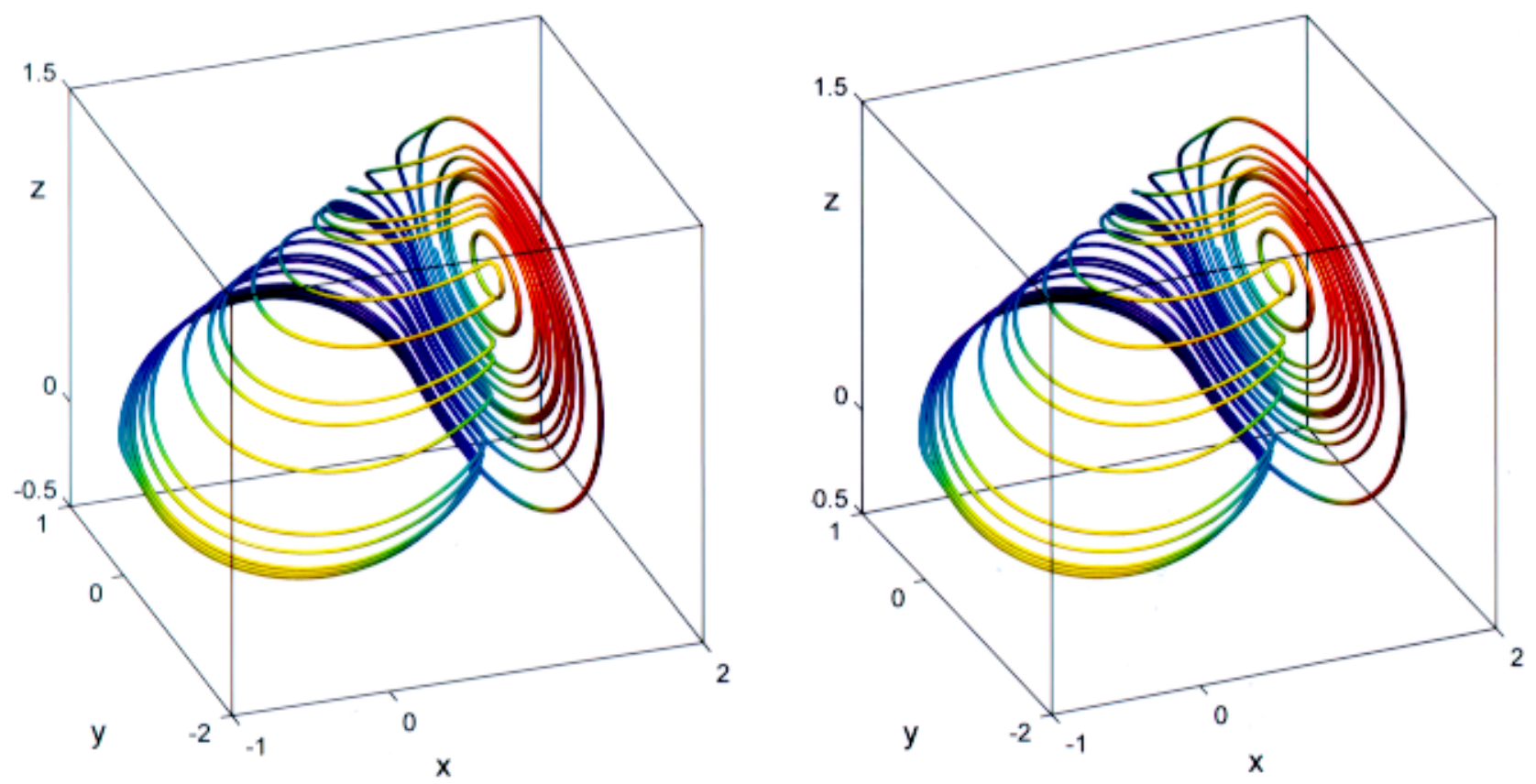

Fig. 13. Stereoscopic view to 3 -D phase-portrait of chaotic attractor. $\quad\left(p_{1}=0.3625 ; p_{2}=1.063125 ; p_{3}=0.2765625\right.$; $q_{1}=-10.2875 ; q_{2}=-1.2 ; q_{3}=-2.71875$.)
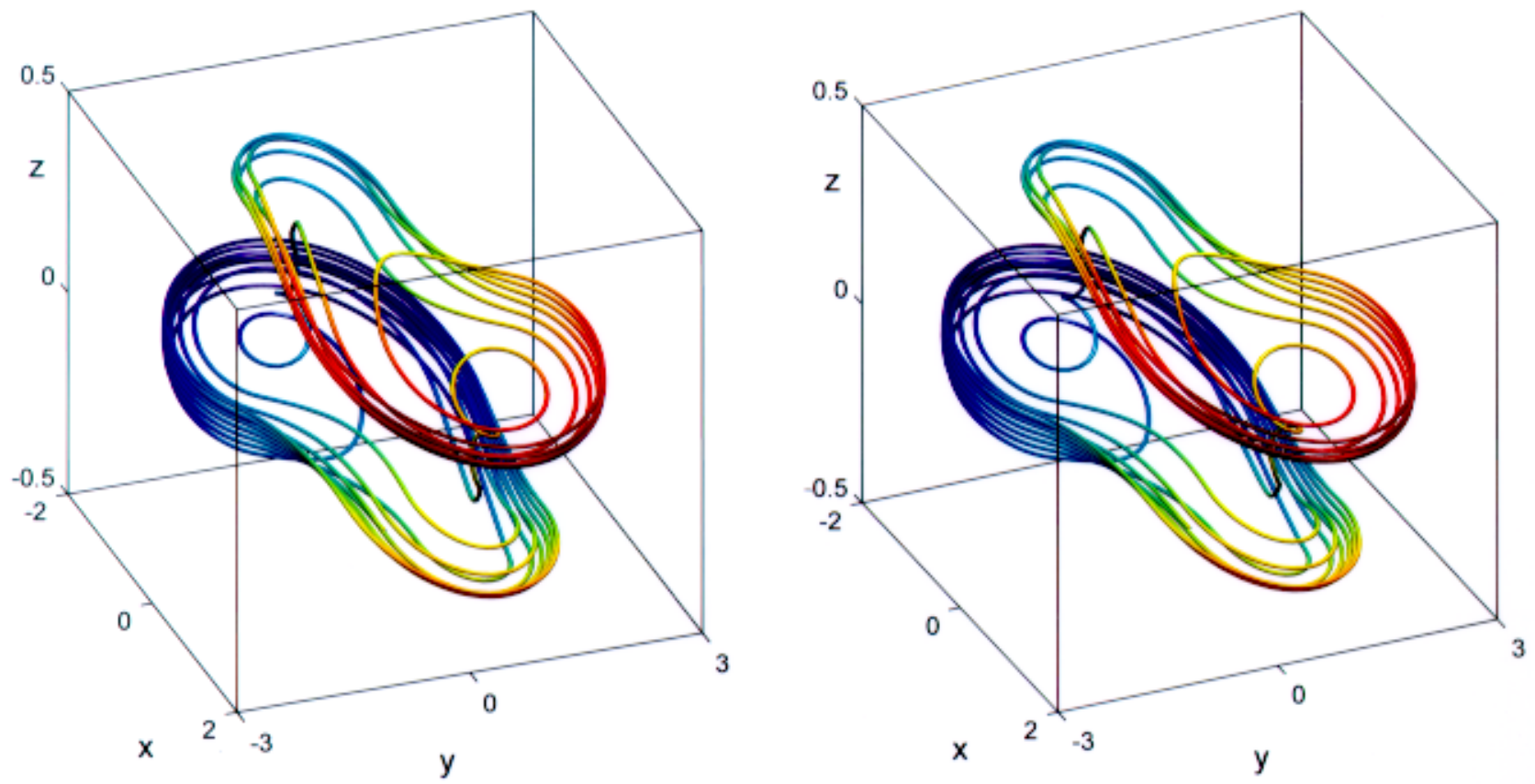

Fig. 14. Stereoscopic view to 3 -D phase-portrait of chaotic attractor. $\quad\left(p_{1}=0.55162 ; p_{2}=-0.21821 ; p_{3}=-0.02724\right.$; $q_{1}=-0.28916 ; q_{2}=0.59760 ; q_{3}=0.00916567$.)

Fig. 11(f) (second canonical form) which indicates the additional reflection around the $y_{\mathrm{II}}-z_{\mathrm{II}}$ plane.

\section{Examples of Typical Attractors}

In this section several examples of typical chaotic attractors represented by the first canonical ODE form are shown. To achieve the best 3-D insight into the chosen model dynamics, a set of trajectories generated by MATLAB in stereoscopic form are introduced in Figs. 12-17. To emphasize the stereo effect the trajectories are displayed in 

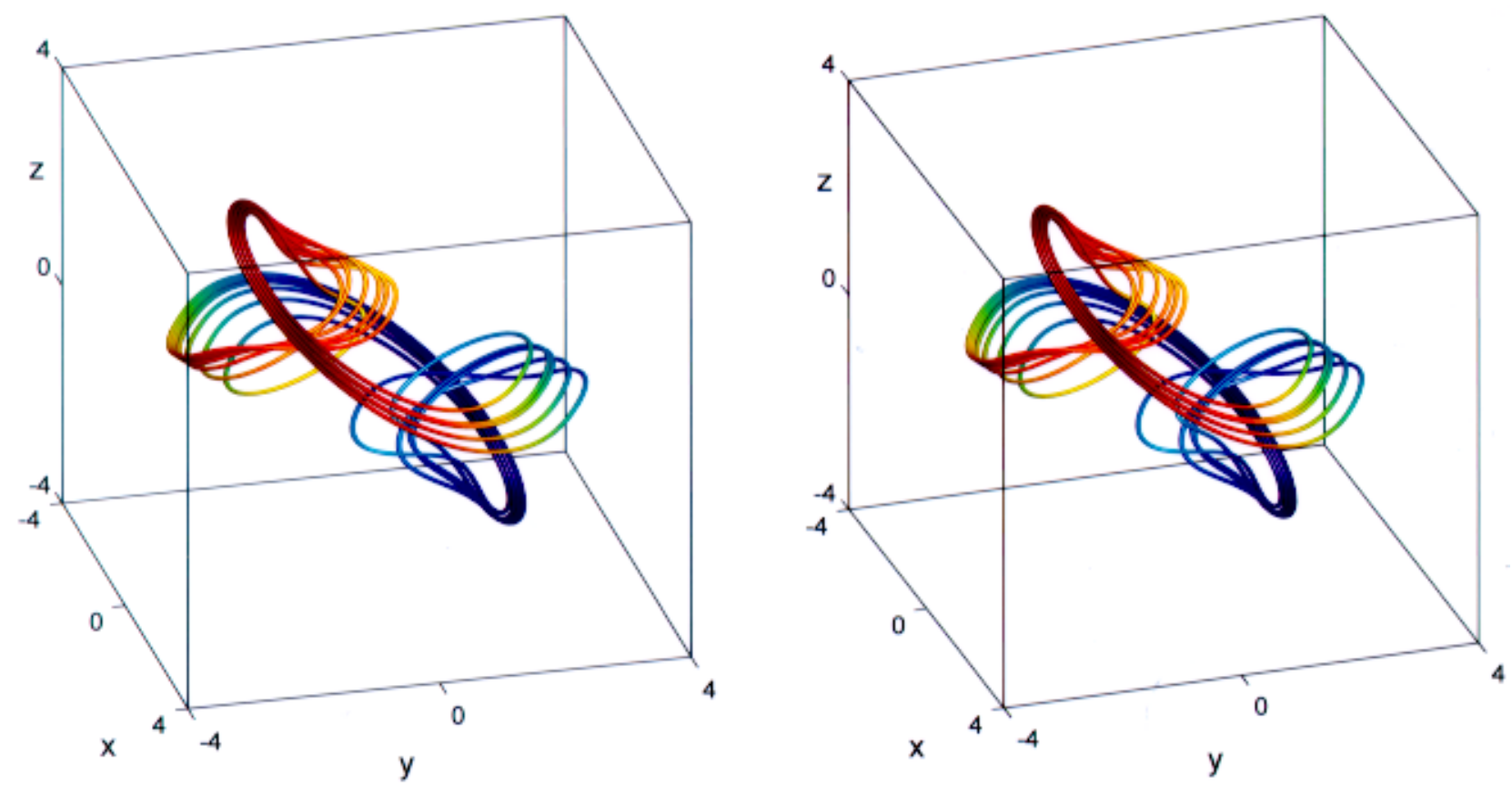

Fig. 15. Stereoscopic view to 3 -D phase-portrait of chaotic attractor. $\left(p_{1}=-1.58383 ; p_{2}=2.67068 ; p_{3}=1.16767\right.$; $q_{1}=0.16767 ; q_{2}=0.91917 ; q_{3}=-2.33534$.)
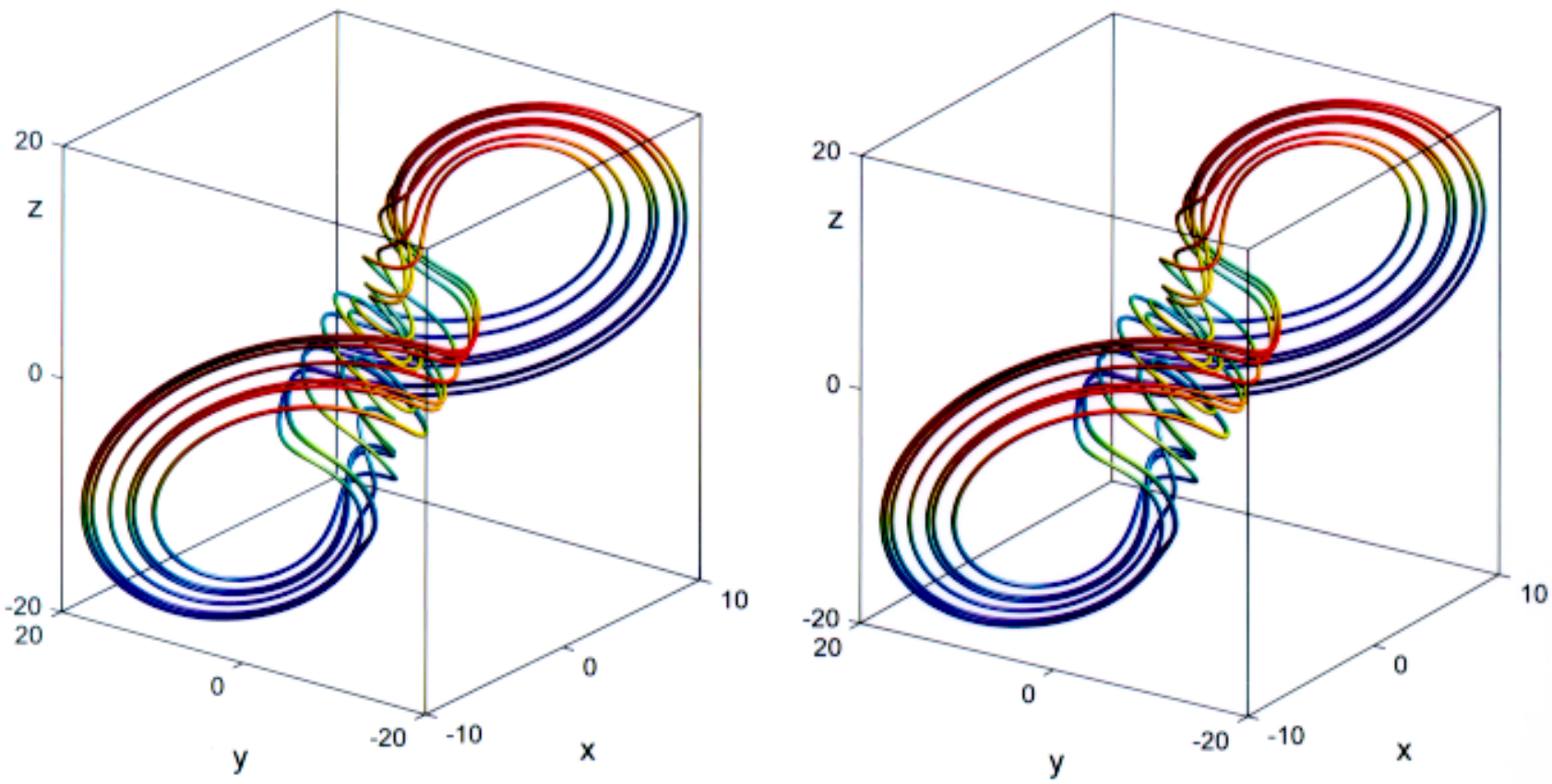

Fig. 16. Stereoscopic view to 3 -D phase-portrait of chaotic attractor. $\left(p_{1}=-0.122 ; p_{2}=11.92958 ; p_{3}=7.655104 ; q_{1}=-1.91\right.$; $\left.q_{2}=0.141025 ; q_{3}=-2.38746.\right)$

reduced number as overlapped "tubes" with optimized diameter. Moreover, different continuously varying colors are used that depend on the sight depth and the illumination from a light source. This produces a little reflection effect on "tubes" and increases the contrast. The details of individual trajectories are given in the corresponding captions.

The instructions on how to view these pairs of trajectories for the best stereoscopic effect are the 

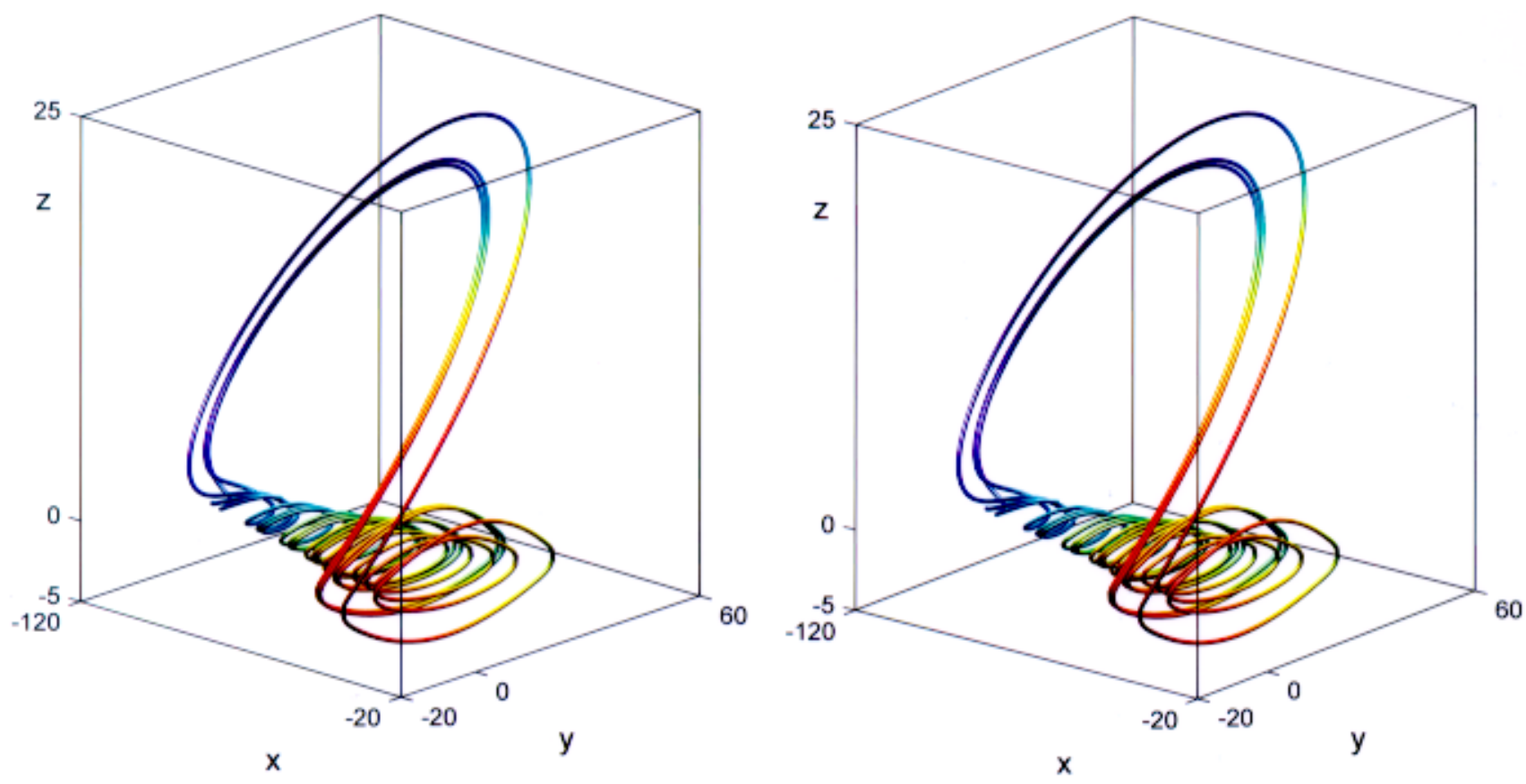

Fig. 17. Stereoscopic view to 3 -D phase-portrait of chaotic attractor. $\left(p_{1}=0.8 ; p_{2}=100.21 ; p_{3}=20.018 ; q_{1}=-2.4\right.$; $q_{2}=-0.71 ; q_{3}=-3.27$.)

following ${ }^{6}$ :

Look at the pair of images perpendicularly from about $50 \mathrm{~cm}$, with ample lighting, and squeeze your eyes slightly in order to see a third image between the two "real" images. The moment you see each of the two images doubled, try to merge the two inner images into one central position. Once you have succeeded in focusing on the third image, you can see it clearly in three dimensions.

\section{Conclusions}

Both canonical ODE forms represent the simplest dual equivalents to Chua's equations and can be used, similarly as Chua's model, in particular for a basic study of the chaotic behavior of all systems belonging to Class $C$ of vector fields in $\Re^{3}$ as well as for the corresponding circuit model realization as possible prototypes. They are topologically conjugate to Class $C$ including the set of measure zero because their equation parameters are given directly by the equivalent eigenvalue parameters. Unlike Chua's equations they are not suitable for direct model- ing of bifurcation phenomena. All these three ODE equivalents, i.e. Chua's equations and both canonical ODE forms, therefore, represent a complete set of mutual complementary models which is useful for modeling, simulation and practical circuit realization of the third-order PWL chaotic systems. In the next paragraph, a brief survey of the latest results based on canonical ODE equivalents are summarized.

The basic third-order forms [Pospíšil et al., 1995; Pospíšil \& Brzobohatý, 1996a] have been extended for higher-order systems [Pospíšil \& Brzobohatý, 1996b] and generalized also for the multiple-feedback PWL function [Pospíšil et al., 1997b]. The utilization of these models for the synchronized chaos simulation has been described in [Pospíšil et al., 1997a; Pospíśil et al., 1999d], and their expression in a complex form is suggested in [Pospísil et al., 1998]. Mutual relations between nonautonomous linear and autonomous PWL systems and the corresponding state models are discussed in [Pospíśil et al., 1999a] and generalized in [Pospíšil et al., 1999e]. Some new models of third-order systems based on the state matrix block decomposition are introduced in [Pospíšil et al.,

\footnotetext{
${ }^{6}$ Note that all the stereoscopic pictures shown in Figs. 12-17 are designed for such final viewing where the right/left eye is focused on the left/right image, respectively. For another possibility, where direct viewing is supposed, i.e. right to right and left to left, it would be necessary to change the sign of the "divergence" angle between the left and right images.
} 
1999b], their generalization for higher-order systems is given in [Pospíšil et al., 1999c]. A practical analog circuit realization based on the first canonical ODE form is described in [Hanus, 1997]. The digital setting of their parameters and the consequent PC optimization are given in [Kolka \& Michálek, 1996; Hanus, 1998], a suitable improved integrator is described in [Nandi et al., 1994]. Another realization by the CNN technique is introduced in [Kaderka, 1996; Švajda \& Kaderka, 1998]. The first results of sensitivity analysis of canonical ODE forms are given in [Horská, 1999].

In addition to these latest results some new ones can be expected in the near future especially in the field of geometrical aspects of the linear topological conjugacy and new simple reference ODE form [Pospísil et al., 2000]. After finishing the comparative study of the eigenvalue sensitivity properties of various ODE forms, the design procedure can be completed in order to obtain their optimized type for individual applications.

\section{Acknowledgments}

The authors would like to thank Prof. V. Špány from the Technical University in Košice for valuable discussions and comments about the concept of canonical ODE equivalents, Dr. K. Kalný from Computer Press Agency, Ltd. for his advice in the preparation of the stereoscopic color pictures including that on the cover, and Dr. S. Hanus for his kind help in the preparation of this manuscript. This work was supported by the Grant Agency of the Czech Republic, Grant No. 102/99/0203 and by the Ministry of Education, Research Programme CEZ: J22/98: 262200011.

\section{References}

Arena, P., Baglio, S., Fortuna, L. \& Manganaro, G. [1995] "Chua's circuit can be generated by CNN cells," IEEE Trans. CAS-42(2), 123-125.

Belykh, V. \& Chua, L. [1992] "New type of strange attractor from a geometric model of Chua's circuit," Int. J. Bifurcation and Chaos 2(3), 697-704.

Brown, R. [1993] "Generalizations of the Chua equations," IEEE Trans. CAS-II40(11), 878-884.

Chua, L., Komuro, M. \& Matsumoto, T. [1986] "The double scroll family, Parts I and II," IEEE Trans. CAS-33(11), 1073-1118.

Chua, L. \& Lin, G. [1990] "Canonical realization of Chua's circuit family," IEEE Trans. CAS-37(6), 885-902.
Chua, L. [1992] "The genesis of Chua's circuit," Archiv für Elektronik und Übertragungs technik 46(4), 250-257.

Chua, L. [1993] "Global unfolding of Chua's circuit," IEICE Trans. Fundamentals E76-A(5), 704-734.

Chua, L., Wu, W., Huang, A. \& Zhong, G. [1993a] "A universal circuit for studying and generating chaos Part I: Routes to chaos," IEEE Trans. CAS-I40(10), 732-744.

Chua, L., Wu, W., Huang, A. \& Zhong, G. [1993b] "A universal circuit for studying and generating chaos - Part II: Strange attractors," IEEE Trans. CASI40(10), 745-761.

Cruz, J. \& Chua, L. [1992] "A CMOS IC nonlinear resistor for Chua's circuit," IEEE Trans. CAS-I39(12), 985-995.

Delgado-Restituto, M. \& Rodriguez-Vasquez, A. [1998] "Design consideration for integrated continuoustime chaotic oscillators," IEEE Trans. CAS-I45(4), 481-495.

Feldmann, U., Hasler, M. \& Schwarz, W. [1996] "Communication by chaotic signals: The inverse approach," Int. J. Circuit Th. Appl. 25(4), 551-579.

Hanus, S. [1997] "Realization of the third-order chaotic systems using their elementary canonical circuit models," Proc. "Rádioelektronika' 97" Bratislava, pp. $44-45$.

Hanus, S. [1998] "Elementary canonical state models using digital gains control," Proc. "Radioelektronika' 98" Brno (2), pp. 352-354.

Hasler, M. [1994] "Synchronization principles and applications," Circuits Syst.: Tutorials IEEE-ISCAS'94, pp. $314-326$.

Horská, J. [1999] "Sensitivity analysis of elementary canonical state models of PWL dynamical systems," Proc. "Radioelektronika' 99" Brno, pp. 360-362.

Huang, A. [1996] "On bounds of the parametric range of bifurcation of Chua's circuit," IEEE Trans. CASI43(8), 691-695.

Huang, A., Pivka, L. \& Wu, C. [1996] "Chua's equation with cubic nonlinearity," Int. J. Bifurcation and Chaos 6, 2175-2222.

Kaderka, J. [1996] "Modelling of third-order elementary canonical state models using cellular neural networks," Proc. ICECS 96 Rhodos, pp. 295-298.

Kahlert, C \& Chua, L. [1990] "A generalized canonical piecewise-linear representation," IEEE Trans. CAS37(3), 373-383.

Kennedy, M. [1992] "Robust op amp realization of Chua's circuit," Frequenz 46(34), 66-80.

Kennedy, M. [1993a] "Three steps to chaos - Part I: Evolution," IEEE Trans. CAS-I40(10), 640-656.

Kennedy, M. [1993b] "Three steps to chaos - Part II: A Chua's circuit primer," IEEE Trans. CAS-I40(10), 657-674.

Kočarev, L., Kardzinov, L. \& Chua, L. [1993] 
"N-dimensional canonical Chua's circuit," J. Circuit Syst. Comput. 3(1), 239-358.

Kolka, Z. \& Michálek, V. [1994] "Digitally controlled linear four-port network," Radioengineering 3(3), 26-28.

Laker, K. \& Ghausi, M. [1974] "Synthesis of lowsensitivity multi-loop feedback active RC filter," IEEE Trans. CAS-21(3), 252-259.

Liao, T. \& Chen, F. [1998] "Control of Chua's circuit with a cubic nonlinearity via nonlinear linearization technique," Circuits Syst. Sign. Process. 17(6), 719-731.

Lindberg, E. [1993] "A note on the modelling and simulation of Chua's circuit," J. Circuit Syst. Comput. 3(1), 537-552.

Madan, R. (Guest ed.) [1993a] "Special issue on Chua's circuit: A paradigm for chaos, Part I," J. Circuit Syst. Comput. 3(1).

Madan, R. (Guest ed.) [1993b] "Special issue on Chua's circuit: A paradigm for chaos, Part II," J. Circuit Syst. Comput. 3(2).

Matsumoto, T. [1984] "A chaotic attractor from Chua's circuit," IEEE Trans. CAS-31(12), 1055-1058.

Nandi, R., Sanyal, S. \& Debroy, S. [1994] "A digitally programmable differential integrator with enlarged time constant," Radioengin. 3(4), 11-17.

Nasser, A., Hosny, E. \& Sobhy, M. [1993] "Maximum dynamic range of bifurcations of Chua's circuit," $J$. Circuit Syst. Comput. 3(1), 471-481.

Neuman, F. [1991] Global Properties of Linear Ordinary Differential Equations (Academia Prague \& Kluwer Academic Public, London).

Ogorzalek, M. [1987] "Chaotic regions from double scroll," IEEE Trans. CAS-34(2), 201-203.

Ogorzalek, M. [1993a] "Taming chaos: Part I — Synchronization," IEEE Trans. CAS-I40(10), 693-699.

Ogorzalek, M. [1993b] "Taming chaos: Part II Control," IEEE Trans. CAS-I40(10), 700-706.

Perry, D. [1975] "New multiple feedback active RC network," Electron. Lett. 11, 364-365.

Pivka, L. \& Špány, V. [1993]. "Boundary surfaces and basin bifurcations in Chua's circuit," J. Circuit Syst. Comput. 3(1), 441-470.

Pospíšil, J., Brzobohatý, J. \& Kolka, Z. [1995] "Elementary canonical state models of the thirdorder autonomous piecewise-linear dynamical systems," Proc. "ECCTD' 95" Istanbul, pp. 463-466.

Pospíšil, J. \& Brzobohatý, J. [1996a] "Elementary canonical state models of Chua's circuit family," IEEE Trans. CAS-I43(8), 702-705.

Pospíšil, J. \& Brzobohatý, J. [1996b] "Canonical state models for higher-order chaos simulation in autonomous piecewise-linear dynamical systems," Int. J. Chaos Th. Appl. 1, 19-25.

Pospíšil, J., Brzobohatý, J. \& Kaderka, J. [1997a] "Utilization of canonical state models for the synchro- nized chaos simulation," Proc. "Rádioelektronika' 97" Bratislava, pp. 40-43.

Pospíšil, J., Brzobohatý, J. \& Hanus, S. [1997b] "Multiple feedback canonical state models of generalized Chua's circuit family," Int. J. Chaos Th. Appl. 2(3\&4), 49-52.

Pospíšil, J., Brzobohatý, J., Hanus, S. \& Michálek, V. [1998] "Complex form of state models of piecewiselinear dynamical systems," Proc. "NOLTA'98" Crans-Montana, (3), pp. 1121-1123.

Pospíšil, J., Hanus, S.-Michálek, V. \& Dostál, T. [1999a] "Relation of canonical state models of linear and piecewise-linear dynamical systems," Proc. $M I C^{\prime} 99$ Innsbruck, pp. 388-389.

Pospíšil, J., Brzobohatý, J., Kolka, Z. \& HorskáKreuzigerová, J. [1999b] "New canonical state models of Chua's circuit family," Radioengin. 8(3), 1-5.

Pospíšil, J., Brzobohatý, J., Kolka, Z. \& HorskáKreuzigerová, J. [1999c] "New canonical state models of the higher-order piecewise-linear dynamical systems," Proc. "NDES' 99" Rønne, pp. 45-48.

Pospíšil, J., Brzobohatý, J. \& Kolka, Z. [1999d] "Elementary canonical state models of Lur'e systems and their application in synchronized chaos," Proc. "ECCTD' 99" Stresa, pp. 867-868.

Pospíšil, J., Hanus, S., Michálek, Brzobohatý, J. \& Horská, J. [1999e] "Generalized relation between linear nonautonomous and piecewise-linear autonomous dynamical systems," Proc. "STO-7" Brno, p. 16.

Pospíšil, J., Brzobohatý, J., Kolka, Z. \& Horská, J. [2000] "New simple reference state model of the third-order piecewise-linear dynamical systems," Radioengineering 9(3), 1-4.

Schwarz, W., Götz, M. \& Feldmann, U. [1993] "Synthesis of higher dimensional Chua circuit," IEEE Trans. CAS-40(11), 854-860.

Schwarz, W. \& Feldmann, U. [1994] "Linear conjugacy of $n$-dimensional piecewise-linear systems," IEEE Trans. CAS-41(2), 190-192.

Švajda, M. \& Kaderka, J. [1998] "Current-mode realization of elementary canonical state models," Proc. "Radioelektronika' 98" Brno, pp. 28-31.

Wu, W. \& Chua, L. [1996] "On linear topological conjugacy of Lur'e systems," IEEE Trans. CAS-I43(2), $158-161$.

Zou, F. \& Nossek, J. [1993] "An autonomous chaotic cellular neural network and Chua's circuit," J. Circuit Syst. Comput. 3(2), 591-601.

Zhong, G. \& Ayrom, F. [1985] "Experimental confirmation of chaos from Chua's circuit," Int. J. Circuit Th. Appl. 13(1), 93-98.

Zhong, G. [1994] "Implementation of Chua's circuit with a cubic nonlinearity," IEEE Trans. CAS-I41(12), 934-940. 